\title{
Precision QCD phenomenology of exotic spin-2 search at the LHC
}

\author{
Goutam Das, ${ }^{a}$ M.C. Kumar ${ }^{b}$ and Kajal Samanta ${ }^{b}$ \\ ${ }^{a}$ Theoretische Physik 1, Naturwissenschaftlich-Technische Fakultät, Universität Siegen, \\ Walter-Flex-Strasse 3, 57068 Siegen, Germany \\ ${ }^{b}$ Department of Physics, Indian Institute of Technology Guwahati, \\ Guwahati-781039, Assam, India \\ E-mail: goutam.das@uni-siegen.de, mckumar@iitg.ac.in, \\ kajal.samanta@iitg.ac.in
}

ABSTRACT: The complete next-to-next-to leading order (NNLO) QCD correction matched with next-to-next-to leading logarithm (NNLL) has been studied for Drell-Yan production via spin-2 particle at the Large hadron collider (LHC). We consider generic spin-2 particle which couples differently to quarks and gluons (non-universal scenario). The threshold enhanced analytical coefficient has been obtained up to third order using the universal soft function and the process dependent form factors at the same order. We performed a detailed phenomenological analysis and gave a prediction for the $13 \mathrm{TeV}$ LHC for the search of such BSM signature. We found that the resummed result gives sizeable corrections over a wide range of invariant mass of the lepton pair. The scale variation also stabilizes at this order and reduces to $4 \%$. As a by-product, we also provide ingredients for third-order soft-virtual (SV) prediction as well as resummation and study the impact on LHC searches.

KeYwords: Phenomenological Models, QCD Phenomenology

ArXiv EPrint: 2011.15121 


\section{Contents}

1 Introduction 1

2 Theoretical framework 3

$\begin{array}{llr}3 & \text { Numerical results } & 8\end{array}$

$\begin{array}{llr}4 & \text { Summary } & 16\end{array}$

$\begin{array}{ll}\text { A Anomalous dimensions } & 17\end{array}$

$\begin{array}{ll}\text { B Process dependent resum coefficients } & 17\end{array}$

C Soft-virtual coefficients at the third order $\quad 22$

\section{Introduction}

Following the discovery of the Higgs boson at the Large Hadron Collider (LHC) [1, 2], much of the focus is given to the search for signals of possible beyond Standard Model (BSM) Physics scenarios. There have been many such models proposed to address various problems in the Standard Model (SM) viz. supersymmetry, extra dimensions, little Higgs, technicolor etc. [3]. These signals can be seen either in the form of smooth deviations from the SM predictions due to contact interactions, or in the form of resonances due to the existence of new heavy particles that these models predict.

In the context of LHC, there have been various observable like invariant mass and transverse momentum of the final states involving either leptons or photons that have a very large detection efficiency $(\geq 90 \%)$ at the ATLAS and CMS detectors. Among such observable, the dilepton invariant mass is of particular interest as the lepton (electron and muon) signals are very clean and the invariant mass $(Q)$ of the lepton pair has been measured up to $2 \mathrm{TeV}$ to a very good accuracy [4-6]. It is to be noted that with the availability of such increasing precision in the experimental data, an adequate theoretical prediction to that accuracy is necessary. After the computation of the Higgs production to $\mathrm{N}^{3} \mathrm{LO}$ accuracy [7], such a high precision has been achieved for the dilepton production at hadron colliders, thanks to the recent computation in the photon mediated channel [8] and the massive gauge boson $W^{ \pm}$production [9]. Such a precision both in the theoretical as well as in the experimental frontiers facilitates a robust search for BSM signals.

In this work, we focus on one particular BSM scenario where generic massive spin-2 fields interact with the SM ones. Such a model is well motivated in the context of search for spin-2 particles (graviton) that couple to SM bosons and fermions differently. The phenomenology of such a graviton is similar to some extent to that of the RS model [10] 
where the massive graviton couples with equal strength (universal) to fermions and bosons in the SM. However unlike the RS model, the parameter space of the non-universal case is much flexible and less constrained at the LHC searches so far. In the context of Higgs characterisation, this model has been studied extensively in the di-boson channels [11]. Later, a complete automation has been done at NLO [12] in the FEYNRuLES [13]MADGRAPH5_AMC@NLO [14] framework. A detailed phenomenology has been performed there for arbitrary values of mass of graviton and its couplings to the SM including parton shower effects as well. It has been observed that the K-factors for different channels give sizeable contribution that depend on the choice of model parameters as well as phase space region. This necessitates further higher order QCD corrections for this model. The first complete next-to-next-to leading order (NNLO) computation has been performed [15] in the dilepton channel. A detailed analysis has been presented there along with the comparison with universal scenario. It has been observed that the K-factors at NLO and NNLO are significantly different from those of the ADD [16, 17] or RS [10] cases. Note that both in $\mathrm{ADD}$ and $\mathrm{RS}$ models, the K-factors for the dilepton production are found to be much larger than those in the SM. This behaviour is well understood because of the presence of additional gluon fusion process (Higgs-like) at the born level in addition to the quark annihilation process (Drell-Yan-like) for graviton production. Such a gluon fusion process at $\mathrm{LO}$ is absent in the SM and the vector boson production takes place via only quark annihilation process. In the present model, the graviton production takes place at the LO via Higgs-like and DY-like processes similar to the ADD or RS model, but with different couplings to quarks $\left(k_{q}\right)$ and gluons $\left(k_{g}\right)$. This particular feature of this model controls the size of the contributions from different channels at higher orders for different choices of model parameters.

The observed discrepancy between the K-factors for the graviton production in the universal and the non-universal cases can be explained to some extent with the help of additional parton radiations at higher orders. For example, a DY-like process with small $k_{q}$ coupling can receive large corrections when the graviton couples with large coupling $k_{g}$ to gluon emitted from the quark lines at higher orders. Similar is the case for Higgslike graviton process. The contribution of other parton level subprocesses initiated by $q g$ starting from NLO and $q q$ ' from NNLO onwards will have further noticeable contributions. For example at NLO, the $q \bar{q}$ subprocess gets corrections a few times larger than the LO subprocess for the choice of parameters where $k_{q} \ll k_{g}$ simply due to the fact that high $k_{g}$ contribution appear at NLO from $q \bar{q}$ subprocess itself. The contribution from the $q g$ channel could be as large as that one for $q \bar{q}$ channel for different parameter choices. The presence of such model dependent large contributions at higher orders for an inclusive process can not be estimated with a simple scaling of the lower order cross sections with the conventional (NLO/NNLO) K-factors computed either in the SM or in the RS model. This motivates not only a detailed phenomenological study at higher orders in QCD but also questions the convergence of the perturbation theory. This is particularly important in the higher mass region where one would normally expect the BSM effect. This region receives large corrections from threshold logarithms and needs a procedure to resum them to all orders. The resummation procedure has been pioneered [18-25] over the last few 
decades to systematically include those large contributions in the threshold region from all orders for better predictions of the perturbative theory.

At the LHC energies where the parton fluxes are large, the threshold logarithms can give sizeable contribution and through resummation these lead to better perturbative convergence as well as theoretical uncertainties for various processes [20, 22, 24, 26-43]. Recently the effect of these large threshold logarithms has been studied in ADD and RS models $[44,45]$ and better perturbative results have been predicted at $\mathrm{N}^{3} \mathrm{LL}(\mathrm{NNLL})$ accuracies. In view of this, we systematically include the threshold resummation effects in DY production for non-universal spin-2 model and present a detailed phenomenological results up to NNLL accuracy. Moreover we have also computed the necessary ingredients to perform resummation up to $\mathrm{N}^{3} \mathrm{LL}$.

The paper is organized as follows. In the section 2, we develop the theoretical formalism and collect important formulae needed for DY production at the LHC. In section 3, we present detailed numerical results for the resummed cross-section matched to those in the fixed order at NNLO level. We also present a quantitative estimate of the threshold corrections at the fixed order $\mathrm{N}^{3} \mathrm{LO}$ level and finally give a summary of findings in section 4 .

\section{Theoretical framework}

The interaction of spin-2 field $\left(h_{\mu \nu}\right)$ with the SM ones is described through the following effective action,

$$
S_{\text {int }}=-\frac{1}{2} \int d^{4} x h_{\mu \nu}(x)\left(\hat{k}_{I} \hat{\mathcal{O}}^{I, \mu \nu}(x)\right), \quad I \in\{G, Q\} .
$$

Sum over repeated indices is implied in all equations. The interaction action has been split in a way so that $I=G$ contains purely gauge terms whereas $I=Q$ contains the fermionic sector and its gauge interactions terms. This decomposition however is not unique and one can shuffle gauge invariant terms between these two. Here $\hat{k}_{I}$ are the unrenormalized coupling constants of the spin-2 field to the operators $\hat{\mathcal{O}}^{I, \mu \nu}$. These gauge invariant operators have the following expressions in terms of unrenormalized quarks and gluon fields, ${ }^{1}$

$$
\begin{aligned}
\hat{\mathcal{O}}_{\mu \nu}^{Q}= & \frac{i}{4} \overline{\hat{\psi}}\left\{\gamma_{\mu} \overrightarrow{D_{\nu}}-\overleftarrow{D_{\nu}} \gamma_{\mu}\right\} \hat{\psi}-\frac{i}{2} g_{\mu \nu} \hat{\psi} \gamma^{\sigma} \overrightarrow{D_{\sigma}} \hat{\psi}+(\mu \leftrightarrow \nu) \\
\hat{\mathcal{O}}_{\mu \nu}^{G}= & \frac{1}{4} g_{\mu \nu} \hat{F}_{\rho \sigma}^{a} \hat{F}^{a, \rho \sigma}-\hat{F}_{\mu \rho}^{a} \hat{F}_{\nu}^{a, \rho}-\frac{1}{\hat{\xi}} g_{\mu \nu} \partial^{\rho}\left(\hat{A}_{\rho}^{a} \partial^{\sigma} \hat{A}_{\sigma}^{a}\right)-\frac{1}{2 \hat{\xi}} g_{\mu \nu}\left(\partial_{\rho} \hat{A}^{a, \rho}\right)\left(\partial_{\sigma} \hat{A}^{a, \sigma}\right) \\
& +\left\{\frac{1}{\hat{\xi}} \hat{A}_{\mu}^{a} \partial_{\nu}\left(\partial^{\rho} \hat{A}_{\rho}^{a}\right)+\partial_{\mu} \overline{\hat{\omega}}\left(\partial_{\nu} \hat{\omega}^{a}-\hat{g}_{s} f^{a b c} \hat{A}_{\nu}^{c} \hat{\omega}^{b}\right)-\frac{1}{2} g_{\mu \nu} \partial_{\rho} \overline{\hat{\omega}}^{a}\left(\partial^{\rho} \hat{\omega}^{a}-\hat{g}_{s} f^{a b c} \hat{A}_{c, \rho} \hat{\omega}^{b}\right)\right. \\
& \quad+\mu \leftrightarrow \nu\}
\end{aligned}
$$

where $\overrightarrow{D_{\mu}}=\overrightarrow{\partial_{\mu}}-i \hat{g}_{s} T^{a} \hat{A}_{\mu}^{a}$. $\hat{\omega}$ and $\hat{\xi}$ are the ghost field and gauge fixing parameter respectively. Notice that the sum of these operators are protected against radiative corrections

\footnotetext{
${ }^{1}$ Throughout this article we use hat $\left({ }^{\wedge}\right)$ symbol for bare quantities and without hat for renormalized quantities.
} 
to all orders due to the fact that spin-2 fields couple to the conserved energy momentum tensor of SM fields. However individually they are not conserved and requires additional UV renormalization. These operators are closed under UV renormalization and hence renormalization can be performed $[46,47]$ through a mixing matrix $Z$.

The unrenormalized operators $\hat{\mathcal{O}}_{I}^{\mu \nu}(x)$ can be renormalized in a closed form $[46,47]$ using renormalization matrix $Z_{I J}$ as

$$
\mathcal{O}_{I}=Z_{I J} \hat{\mathcal{O}}_{J}
$$

We use dimensional regularization in $d=4-2 \epsilon$ dimensions to regulate both UV and IR divergences. In $d$-dimensions, the bare strong coupling constant can be related to the renormalized one as

$$
\hat{a}_{s} S_{d}=a_{s}\left(\mu_{r}\right) Z\left(a_{s}\left(\mu_{r}\right)\right)\left(\frac{\mu_{r}^{2}}{\mu^{2}}\right)^{\frac{4-d}{2}},
$$

where $S_{d}$ is the spherical factor. Here $Z\left(a_{s}\left(\mu_{r}\right)\right)$ is the strong coupling renormalization constant which takes the following form in $d=4-2 \epsilon$ dimensions,

$$
\begin{aligned}
Z\left(a_{s}\left(\mu_{r}\right)\right)= & 1+a_{s}\left(\mu_{r}\right)\left\{-\beta_{0} \epsilon^{-1}\right\}+a_{s}^{2}\left(\mu_{r}\right)\left\{\beta_{0}^{2} \epsilon^{-2}-\frac{\beta_{1}}{2} \epsilon^{-1}\right\} \\
& +a_{s}^{3}\left(\mu_{r}\right)\left\{-\beta_{0}^{3} \epsilon^{-3}+\frac{7}{6} \beta_{0} \beta_{1} \epsilon^{-2}-\frac{1}{3} \beta_{2} \epsilon^{-1}\right\} .
\end{aligned}
$$

The UV renormalization constants $Z_{I J}$ satisfy the following RGE

$$
\frac{d}{d \ln \mu_{r}} Z_{I J}=\gamma_{I K} Z_{K J}
$$

where $\gamma_{I J}$ are the UV anomalous dimensions.

The interation action can be expressed in terms of renormalized quatities as well i.e. in terms of renormalized couplings $\left(\kappa_{I}\right)$ and renormalized operators $\left(\mathcal{O}_{I}\right)^{2}$ as

$$
S_{\mathrm{int}}=-\frac{1}{2} \int d^{4} x h_{\mu \nu}(x)\left(\kappa_{I} \mathcal{O}_{I}^{\mu \nu}(x)\right) .
$$

The unrenormalized coupling constants are then related to the renormalized ones with the transpose of the same renormalization constants ${ }^{3} Z_{I J}$ as

$$
\hat{\kappa}_{I}=Z_{J I} \kappa_{J} .
$$

The solution of UV renormalization constants RGE (eq. (2.7)) matrix leads to the following expansion in terms of renormalized strong coupling constant up to the third order,

$$
\begin{aligned}
Z_{I J}=\delta_{I J}+a_{s}\left(\mu_{r}\right)\left\{\left(-\gamma_{I J}^{(1)}\right) \epsilon^{-1}\right\}+a_{s}\left(\mu_{r}\right)^{2}\left\{\left(\frac{1}{2} \beta_{0} \gamma_{I J}^{(1)}+\frac{1}{2} \gamma_{I K}^{(1)} \gamma_{K J}^{(1)}\right) \epsilon^{-2}+\left(-\frac{1}{2} \gamma_{I J}^{(2)}\right) \epsilon^{-1}\right\} \\
+a_{s}\left(\mu_{r}\right)^{3}\left\{\left(-\frac{1}{3} \beta_{0}^{2} \gamma_{I J}^{(1)}-\frac{1}{2} \beta_{0} \gamma_{I K}^{(1)} \gamma_{K J}^{(1)}-\frac{1}{6} \gamma_{I K}^{(1)} \gamma_{K L}^{(1)} \gamma_{L J}^{(1)}\right) \epsilon^{-3}+\left(\frac{1}{3} \beta_{1} \gamma_{I J}^{(1)} \frac{1}{3} \beta_{0} \gamma_{I J}^{(2)}\right.\right. \\
\left.\left.+\frac{1}{6} \gamma_{I K}^{(1)} \gamma_{K J}^{(2)}+\frac{1}{3} \gamma_{I K}^{(2)} \gamma_{K J}^{(1)}\right) \epsilon^{-2}+\left(-\frac{1}{3} \gamma_{I J}^{(3)}\right) \epsilon^{-1}\right\} .
\end{aligned}
$$

\footnotetext{
${ }^{2}$ Note that in a pure gauge theory $\left(n_{f}=0\right)$, the operator $\mathcal{O}_{G}^{\mu \nu}$ is conserved.

${ }^{3}$ We confirmed a typo in eq. (2.11) of [48] which however finally does not affect the results there.
} 
All the relevant anomalous dimensions $\left(\gamma_{I J}\right)$ are extracted to three loops [15] from the bare quark and gluon form factors and by simply claiming the universality of the infrared divergences at the same order. For the sake of completeness we have collected those in the appendix A. Note that one can equivalently perform coupling constant renormalization using eq. (2.9) instead of operator renormalization in eq. (2.4) to remove the UV divergences from the form factor.

We now turn our discussion into the DY production cross-section at the LHC which takes the following form in terms of partonic coefficient function $\Delta^{I}$ and luminosity $\mathcal{L}$,

$$
\begin{aligned}
2 S \frac{d \sigma}{d Q^{2}}\left(\tau, Q^{2}\right)= & \sum_{a b=\{q, \bar{q}, g\}} \int_{0}^{1} d x_{1} \int_{0}^{1} d x_{2} \int_{0}^{1} d z \mathcal{L}_{a b}\left(x_{1}, x_{2}, \mu_{f}^{2}\right) \\
& \times \sum_{I} \mathcal{F}_{I}^{(0)} \Delta_{a b}^{I}\left(z, Q^{2}, \mu_{f}^{2}\right) \delta\left(\tau-z x_{1} x_{2}\right) .
\end{aligned}
$$

The luminosity function consists of non-perturbative parton distribution functions. The prefactor $\mathcal{F}_{I}^{(0)}$ is given for SM and for spin-2 (denoted as GR) channels as follows

$$
\begin{aligned}
\mathcal{F}_{\mathrm{SM}}^{(0)}=\frac{4 \alpha^{2}}{3 Q^{2}}\left[Q_{q}^{2}-\frac{2 Q^{2}\left(Q^{2}-M_{Z}^{2}\right)}{\left(\left(Q^{2}-M_{Z}^{2}\right)^{2}+M_{Z}^{2} \Gamma_{Z}^{2}\right) c_{w}^{2} s_{w}^{2}} Q_{q} g_{e}^{V} g_{q}^{V}\right. & \\
& \left.\quad+\frac{Q^{4}}{\left(\left(Q^{2}-M_{Z}^{2}\right)^{2}+M_{Z}^{2} \Gamma_{Z}^{2}\right) c_{w}^{4} s_{w}^{4}}\left(\left(g_{e}^{V}\right)^{2}+\left(g_{e}^{A}\right)^{2}\right)\left(\left(g_{q}^{V}\right)^{2}+\left(g_{q}^{A}\right)^{2}\right)\right] . \\
\mathcal{F}_{\mathrm{GR}}^{(0)}= & \frac{k_{q}^{2} Q^{6}}{80 \pi^{2} \Lambda^{4}}\left|D\left(Q^{2}\right)\right|^{2} .
\end{aligned}
$$

Here $\alpha$ represents the fine structure constant, $c_{w}, s_{w}$ are the sine and cosine of the Weinberg angle and $M_{Z}$ and $\Gamma_{Z}$ are the mass and width of the $Z$ boson respectively. The vector and axial coupling of the weak boson is given as

$$
g_{a}^{A}=-\frac{1}{2} T_{a}^{3}, \quad g_{a}^{V}=\frac{1}{2} T_{a}^{3}-s_{w}^{2} Q_{a},
$$

$\Lambda$ in eq. (2.13) is the cut-off scale of the spin-2 theory which is of $\mathcal{O}(\mathrm{TeV})$ and $k_{I}$ are introduced for convenience and are defined as $k_{I}=\sqrt{2} \kappa_{I} / \Lambda$. The propagator for GR theory with mass $M_{G}$ is given as

$$
D\left(Q^{2}\right)=\frac{1}{\left(Q^{2}-M_{G}^{2}\right)+i \Gamma_{G} M_{G}} .
$$

The analytical results for the partonic coefficient functions are known for some time to the second order for all subprocesses [17]. The partonic coefficients can be decomposed into the following form for the gluon and quark-antiquark initiated processes:

$$
\Delta_{a b}^{I}=\Delta_{a b, s v}^{I}+\Delta_{a b, r e g}^{I}, \quad a b \in\{g g, q \bar{q}\} .
$$

The first term in the above equation is denoted as the soft-virtual (SV) term which consists of form factor contribution as well as soft gluon radiation. The perturbative expansion of the SV coefficients are given as,

$$
\Delta_{a b, s v}^{I}=\sum_{i=0} a_{s}^{i} \mathcal{N}_{a b}^{I} \Delta_{a b, s v}^{(i), I}
$$


$\mathcal{N}_{I}$ are some overall prefactors taken out from the SV coefficient. The $\mathcal{N}_{I}$ prefactors are given as

$$
\begin{aligned}
\mathcal{N}_{a b}^{\mathrm{SM}} & =\frac{2 \pi}{N_{c}}, \\
\mathcal{N}_{q \bar{q}}^{\mathrm{GR}} & =\frac{\pi k_{q}^{2}}{8 N_{c}}, \\
\mathcal{N}_{g g}^{\mathrm{GR}} & =\frac{\pi k_{g}^{2}}{2\left(N_{c}^{2}-1\right)} .
\end{aligned}
$$

Note that with this normalisation in eq. (2.18), the SV coefficient at LO simply becomes $\delta(1-z)$. The main ingredients to compute the SV coefficients (after the PDF renormalization) are the form factor and universal soft distribution functions. As mentioned earlier, the form factors are available in the literature up to three loops [48]. We have used the eq. (2.9) to remove the UV divergences from the bare form factors. The UV finite form factor then contains the IR divergences originating from the soft-collinear region. After performing collinear renormalization of parton distribution functions, the remaining IR divergences are cancelled upon inclusion of universal soft distributions. The universal soft distributions are already known in the literature up to three loops [24, 49-52]. This finally leads to finite SV coefficients up to third order which contains $\delta(1-z)$ and plus-distributions $\mathcal{D}_{i}=\left[\frac{\ln ^{i}(1-z)}{1-z}\right]_{+}$(where at the order $n, i$ can be maximum $2 n-1$ ) in partonic threshold variable $z=Q^{2} / \hat{s}$. The new third order SV coefficients are collected in the appendix C.

In order to better describe the fixed order cross-section particularly in the threshold region, one needs to resum threshold enhanced logarithms to all orders. In the threshold region the partonic $z \rightarrow 1$ induces large singular terms from delta function and plus distributions. The resummation is performed in Mellin space where the threshold limit $z \rightarrow 1$ translates into $N \rightarrow \infty$ and the large logarithms appear in the form of $\ln ^{i} \bar{N}$ (with $\bar{N}=N \exp \left(\gamma_{E}\right)$ and $\gamma_{E}$ is Euler-Mascheroni constant.). The partonic coefficient takes the following exponential form (up to normalisation by born factor.)

$$
\left(d \hat{\sigma}_{N}^{I} / d Q\right) /\left(d \hat{\sigma}_{\mathrm{LO}}^{I} / d Q\right)=g_{0, p}^{I} \exp \left(G_{\bar{N}}^{I}\right)
$$

The born normalisation factor is given as

$$
\begin{aligned}
\left(d \hat{\sigma}_{\mathrm{LO}}^{I} / d Q\right) & =\mathcal{F}_{\mathrm{SM}}^{(0)} \frac{Q}{S}\left\{\frac{2 \pi}{N_{c}}\right\} & & \text { for SM, } \\
& =\mathcal{F}_{\mathrm{GR}}^{(0)} \frac{Q}{S}\left\{\frac{\pi k_{q}^{2}}{8 N_{c}}, \frac{\pi k_{g}^{2}}{2\left(N_{c}^{2}-1\right)}\right\} & & \text { for }\{q \bar{q}, g g\} \text { in } \mathrm{GR} .
\end{aligned}
$$

The prefactor $g_{0, p}^{I}$ can be expanded in terms of strong coupling as

$$
g_{0, p}^{I}=1+\sum_{i=1} a_{s}\left(\mu_{r}\right)^{i} g_{0, p}^{(i), I}, \quad p \in\{q, g\}
$$


The universal resummed exponent in eq. (2.19) can be found from the Mellintransform of the well known universal cusp anomalous dimensions $A_{p}^{I}$ [53-67] and constant $D_{p}^{I}[20,22,44]$,

$$
G_{\bar{N}}^{I}=\int_{0}^{1} d z \frac{z^{N-1}-1}{1-z}\left[\int_{\mu_{f}^{2}}^{Q^{2}(1-z)^{2}} \frac{d \mu^{2}}{\mu^{2}} 2 A_{p}^{I}\left(a_{s}\left(\mu^{2}\right)\right)+D_{p}^{I}\left(a_{s}\left(Q^{2}(1-z)^{2}\right)\right)\right] .
$$

This integration can be performed after expanding the anomalous dimensions in strong coupling constant. The resulting function can be organised in a perturbative series as

$$
G_{\bar{N}}^{I}=\ln \bar{N} g_{1}^{I}(\omega)+g_{2}^{I}(\omega)+a_{s} g_{3}^{I}(\omega)+a_{s}^{2} g_{4}^{I}(\omega)+\cdots,
$$

where $\omega=2 \beta_{0} a_{s} \ln \bar{N}$. Note that in the context of resummation $a_{s} \ln \bar{N} \sim \mathcal{O}(1)$. The successive terms in the above series along with the same for $g_{0, p}^{I}$ in eq. (2.21) define the resummation accuracies LL, NLL etc. The required exponents can be found in $[20,22$, 41, 42]. The coefficients $g_{0, p}^{I}$ which contains the process dependent information can be extracted from the newly computed SV coefficients by just transforming all the $\delta(1-z)$ and plus distributions into the Mellin space and dropping all the $N$-dependent terms. Note that these coefficients differ significantly compared to the universal scenario due to the fact that they now explicitly depend on the couplings. However in the limit when these couplings $\left(k_{q}, k_{g}\right)$ are same, they should reproduce the exact coefficients for the universal case. This serves a crucial check for our computation. We collect these newly computed coefficients up to the third order in the appendix B.

The resummed expression in eq. (2.19) obviously does not contain any contributions which are not enhanced in the threshold limit particularly the regular contributions. These regular pieces from the quark or gluon initiated process or from other subprocesses are also important to describe the full phase space. A consistent way to include those contributions is generally performed through matching procedure given by

$$
\begin{aligned}
{\left[\frac{d \sigma}{d Q}\right]_{N^{n} L O+N^{n} L L}=} & {\left[\frac{d \sigma}{d Q}\right]_{N^{n} L O}+\sum_{a b \in\{q, \bar{q}, g\}} \frac{d \hat{\sigma}_{L O}}{d Q} \int_{c-i \infty}^{c+i \infty} \frac{d N}{2 \pi i}(\tau)^{-N} \delta_{a b} f_{a, N}\left(\mu_{f}^{2}\right) f_{b, N}\left(\mu_{f}^{2}\right) } \\
& \times\left(\left[\frac{d \hat{\sigma}_{N}}{d Q}\right]_{N^{n} L L}-\left[\frac{d \hat{\sigma}_{N}}{d Q}\right]_{t r}\right)
\end{aligned}
$$

The first term on the right hand side is the exact fixed order cross-section at $\mathrm{N}^{n} \mathrm{LO}$. The terms in the parentheses resum all the large logarithms avoiding any double counting from the fixed order counter part. Note that resummation is performed in Mellin space and finally one has to perform a Mellin-inversion. In particular one has to avoid the Landau pole while performing the inversion. This can be done using Minimal prescription [68] or Borel prescription $[69,70]$. We followed the former ${ }^{4}$ approach and fixed the contour accordingly.

Finally we conclude this section with the note that although generally the large Mellin-N logarithms are exponentiated, it is also possible to exponentiate some part or all $g_{0, p}^{I}[32,41,42,72-74]$. However in this article we sticked to the standard approach and studied its effect at the LHC.

\footnotetext{
${ }^{4}$ For a detailed understanding of Borel prescription and comparison with Minimal ones, one can follow e.g. [71].
} 

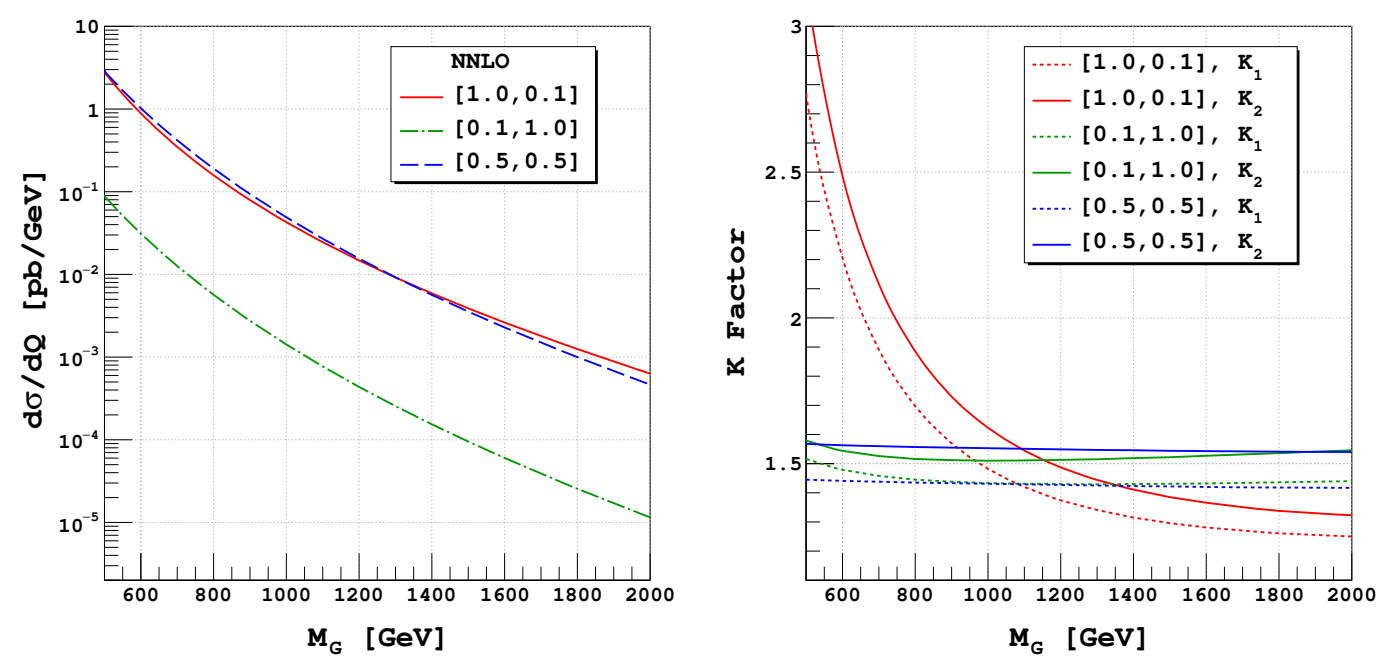

Figure 1. Invariant mass distribution of the dilepton for signal for different choice of $k_{q}$ and $k_{q}$ at $Q=M_{G}$ in NNLO QCD (left panel) and the corresponding NLO and NNLO K-factor as defined eq. (3.1) (right panel).

\section{$3 \quad$ Numerical results}

We now turn to the discussion on numerical result up to NNLO+NNLL accuracy in QCD for dilepton production via spin-2 particle at the LHC. The coupling strength of spin-2 particles to bosons is $\kappa_{G}=\sqrt{2} k_{g} / \Lambda$ and to fermions is $\kappa_{Q}=\sqrt{2} k_{q} / \Lambda$. Here, $\Lambda$, the scale of the theory, is taken to be $3 \mathrm{TeV}$ and we choose $0<\left(k_{q}, k_{g}\right)<1$. For this analysis we use PDF4LHC15 [75] parton distribution functions (PDF) throughout from LHAPDF [76] subroutine unless otherwise stated. For the fixed order as well as resummed computation, we convolute NLO(NNLO) level partonic coefficient functions with NLO(NNLO) PDF. The corresponding strong coupling constant $\alpha_{s}\left(\mu_{r}^{2}\right)$ is also provided by LHAPDF subroutine and for convenience we have defined $a_{s}\left(\mu_{r}^{2}\right)=\alpha_{s}\left(\mu_{r}^{2}\right) /(4 \pi)$. The fine structure constant is taken to be $\alpha_{e m}=1 / 128$ and the weak mixing angle is $\sin ^{2} \theta_{w}=0.22343$. Here we present the results for $n_{f}=5$ flavors in the massless limit of quarks. Our default choice for the center of mass energy of the LHC is $\mathrm{E}_{\mathrm{CM}}=13 \mathrm{TeV}$. Except for the study of scale variations, we set renormalization $\left(\mu_{r}\right)$ and factorization $\left(\mu_{f}\right)$ scales equal to the dilepton invariant mass, i.e. $\mu_{r}=\mu_{f}=Q$.

Before we present the resum result, we discuss some distinctive features of this model that are not observed for the case of universal couplings. For this, in figure 1 left panel we present the dilepton invariant mass distribution at the resonance $Q=M_{G}$ for different choices of the model parameters $k_{q}$ and $k_{g}$. For the results presented just in figure 1, we use NNLO PDF at all orders. We observe that even at the resonance region, the cross section depends on the choice of $k_{q}$ and $k_{g}$, which is not the case for the RS model where the height of the peak is independent of the corresponding coupling $c_{0}$ [45]. This particular nature can be understood from the parton level Born cross sections, eq. (3.2). In figure 1 right 


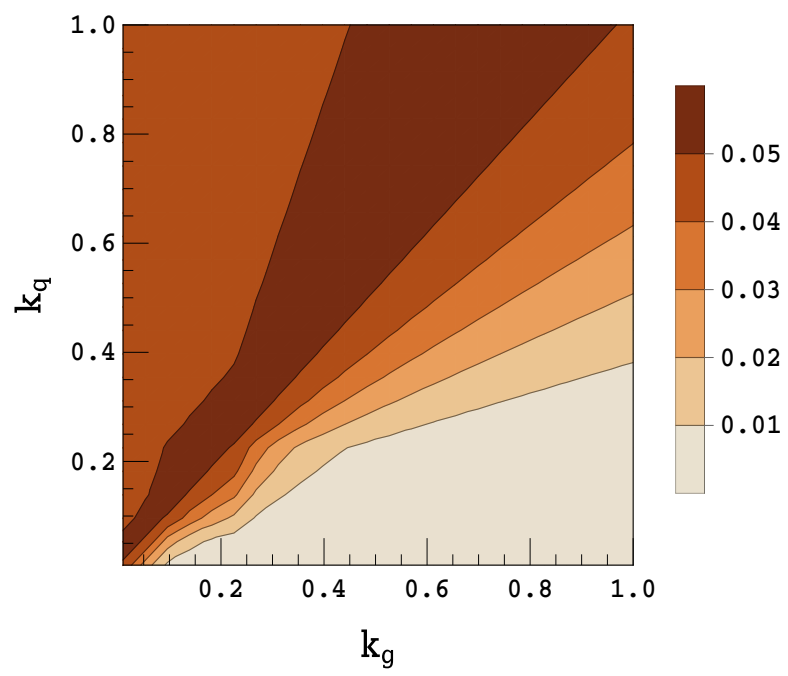

Figure 2. The contour plot of couplings $k_{q}$ and $k_{g}$ for signal at NNLO with a fixed value of the spin-2 mass $M_{G}=1 \mathrm{TeV}$. The plot shows the cross-section (in $p b$ ) at the resonance $Q=M_{G}$.

panel we present the corresponding K-factors $\left(K_{1}, K_{2}\right)$ defined with respect to LO as,

$$
K_{n}=\frac{d \sigma^{N^{n} L O} / d Q}{d \sigma^{L O} / d Q}
$$

Here we observe that for $\left(k_{q}, k_{g}\right)=(1.0,0.1)$ at low $Q=M_{G}$ values, the NLO $K$-factors $K_{1}$ can be as high as 3 while the corresponding $K$-factors for the universal case are about 1.5 [45]. Because $k_{q}$ is much larger than $k_{g}$, at LO one can expect dominant contributions from Drell-Yan like process while the Higgs-like process is suppressed because of smaller coupling $k_{g}$. For the same reason, at NLO the gluon emissions from the underlying born level parton processes is also expected to give smaller contribution. However, at NLO the presence of additional subprocesses like $q g$ with large parton fluxes, give a significant positive contribution comparable to that of $q \bar{q} \mathrm{LO}$ as discussed in the introduction. However for $\left(k_{q}, k_{g}\right)=(0.1,1.0)$ and $(0.5,0.5)$, the $q g$ subprocess contribution is very small and comparable to LO $q \bar{q}$ subprocess and is negative. Because of this potentially large and model dependent contributions at NLO, it is necessary to include the QCD corrections at second order and beyond in the perturbation series. We present the NNLO K-factors $\left(K_{2}\right)$ in the right panel of figure 1 and it can be seen that these second order contributions are smaller than the corresponding NLO ones for different extreme choices of the non-universal couplings and confirms that at NLO all possible dominant contributions are considered giving typical sizeable corrections from NNLO onwards. In this regard, it is convenient to study the higher order QCD corrections in terms of the K-factors $R_{n m}$ defined with respect to NLO. In the rest of the analysis, we present the results in terms of these K-factors $R_{n m}$.

Next, we present the dependence of model parameters $k_{q}$ and $k_{g}$ on the FO cross sections at the resonance for $Q=M_{G}=1 \mathrm{TeV}$ through the contour plot in figure 2 . The cross section is large for larger $k_{q}$ values and becomes maximum around the universal line $\left(k_{q} \sim k_{g}\right)$. The behaviour can be better understood from the dependence of born level 

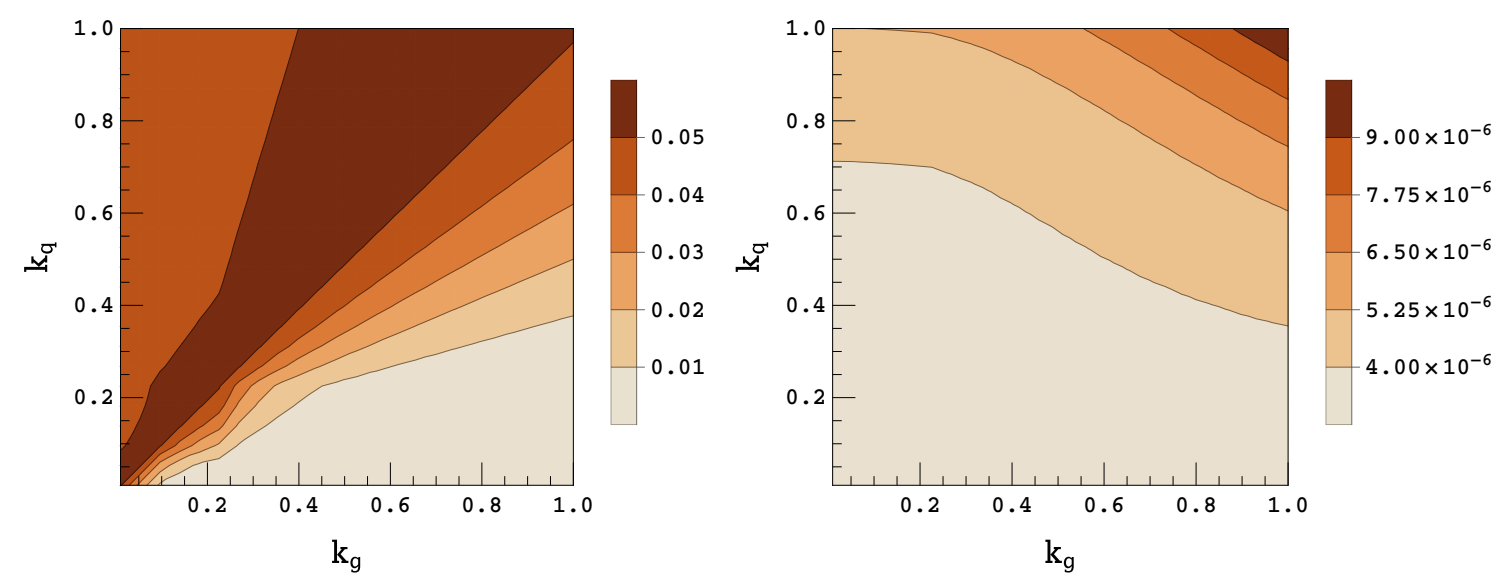

Figure 3. The contour plot of couplings $k_{q}$ and $k_{g}$ for signal at NNLO+NNLL with a fixed value of the spin- 2 mass $M_{1}=1 \mathrm{TeV}$. The left plot shows the cross-section (in $p b$ ) at the resonance $Q=M_{1}$, the right plot shows the cross-section (in $p b$ ) away from the resonance at $Q=1.5 \mathrm{TeV}$.

partonic subprocesses on $k_{q}$ and $k_{g}$ as,

$$
\Delta_{q \bar{q}}^{\mathrm{GR}} \propto \frac{k_{q}^{4}}{C\left(Q^{2}\right)+\left[A k_{q}^{2}+B k_{g}^{2}\right]^{2}}, \quad \Delta_{g g}^{\mathrm{GR}} \propto \frac{k_{q}^{2} k_{g}^{2}}{C\left(Q^{2}\right)+\left[A k_{q}^{2}+B k_{g}^{2}\right]^{2}}
$$

Here the coefficients $A$ and $B$ contain the contributions for the decay of the spin-2 particle to fermions and bosons respectively and $C\left(Q^{2}\right)=\left(Q^{2}-M_{G}^{2}\right)^{2}$. At resonance where $C\left(Q^{2}\right)=0$, the cross section will increase with increasing $k_{q}$ for a fixed $k_{g}$ and $k_{g}$ effect is mild here. For any given $k_{q}$, the cross sections are maximum when $k_{g} \lesssim k_{q}$.

We now discuss the resummed effects for the non-universal couplings. We have performed resummation up to NNLL accuracy and matched them with the fixed order NNLO level as described in section 2. In figure 3, we have shown the dependence of the cross section on $k_{q}$ and $k_{g}$ including the resummation effect at NNLL. In the left panel, we present the contour region at the resonance $Q=M_{G}=1 \mathrm{TeV}$ and in the right panel we present the same but at the off-resonance region $Q=1.5 \mathrm{TeV}$. Note that unlike the RS case, here we find non-negligible GR contribution away from the resonance as well. The dependence on the model parameters is found to be the similar as that observed in the FO case figure 2 . However, the maximum cross section region for the resummed case is wider. We have also studied the behaviour of cross-section by varying the spin-2 mass $M_{G}$ and either of the couplings while keeping the other coupling fixed. In figure 4, we present such behaviour at the resonance region $Q=M_{G}$ to NNLO+NNLL accuracy for different values of $M_{G}$ and $k_{q}$ for a given $k_{g}=1.0$ in the left panel. We observe that with increasing $M_{G}$, for a fixed $k_{q}$ the cross section is falling due to the decreasing parton fluxes. The cross section is increasing with $k_{q}$ for a fixed value of $M_{G}$ as discussed in eq. (3.2). In the right panel, we present similar results but by varying $M_{G}$ and $k_{g}$ for a given value of $k_{q}=1.0$ and we observe the mild effect of $k_{g}$ on cross section when both $M_{G}$ and $k_{q}$ are fixed. 

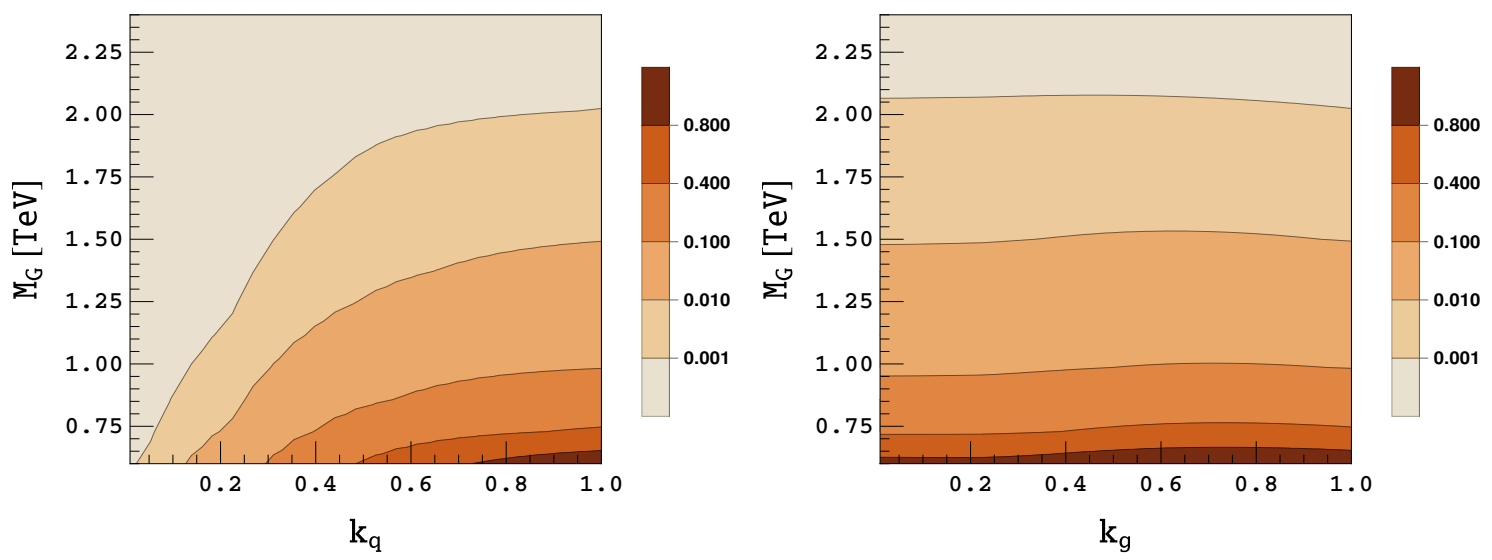

Figure 4. The contour plot for mass of the spin-2 particle with coupling $k_{q}$ and $k_{g}$ for signal at NNLO+NNLL at $Q=M_{G}$. The left panel shows the cross section (in $p b$ ) for different $M_{G}$ and $k_{q}$ region while $k_{g}=1.0$ and the right panel shows the cross section for $M_{G}$ and $k_{g}$ region with $k_{q}$ fixed at 1.0 .

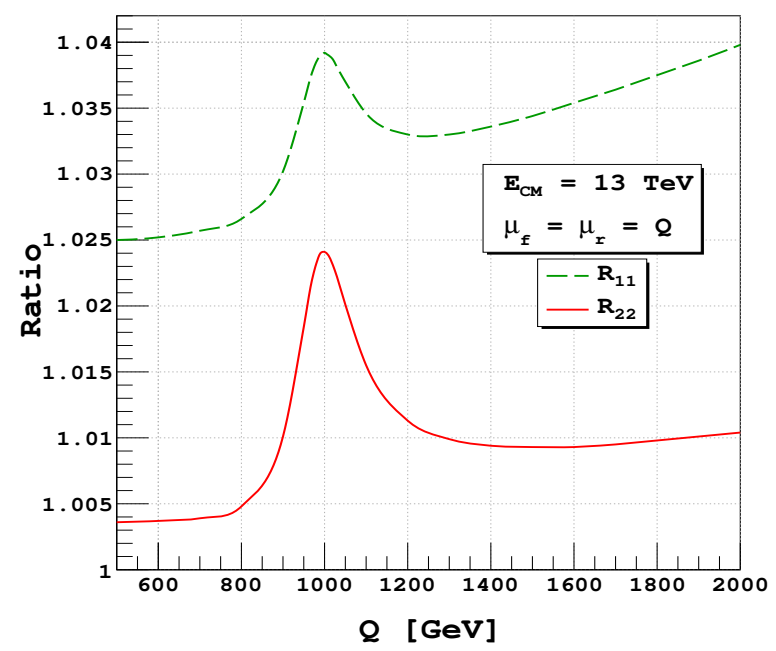

Figure 5. Comparison between fixed order results and resummed results. The ratios are defined with respect to NLO as described in the text.

To quantify the resummation effect we define K-factor with respect to NLO as

$$
R_{n m}=\frac{d \sigma^{\left(N^{n} L O+N^{n} L L\right)} / d Q}{d \sigma^{\left(N^{m} L O\right)} / d Q} .
$$

We present in figure 5, the ratio of resum results to the FO ones as given in eq. (3.3). We notice that there is around $4 \%$ enhancement due to NLL resummation over the NLO FO result at the resonance region while the enhancement due to NNLL resummation is around $2.5 \%$ over the NNLO results. In general we notice that in the region $Q>M_{G}$, the resummation effects keep increasing with $Q$ and are dominant at NLO+NLL level. However at NNLO+NNLL this is almost constant and gives only $1 \%$ increment over the NNLO in the region $Q>M_{G}$. 

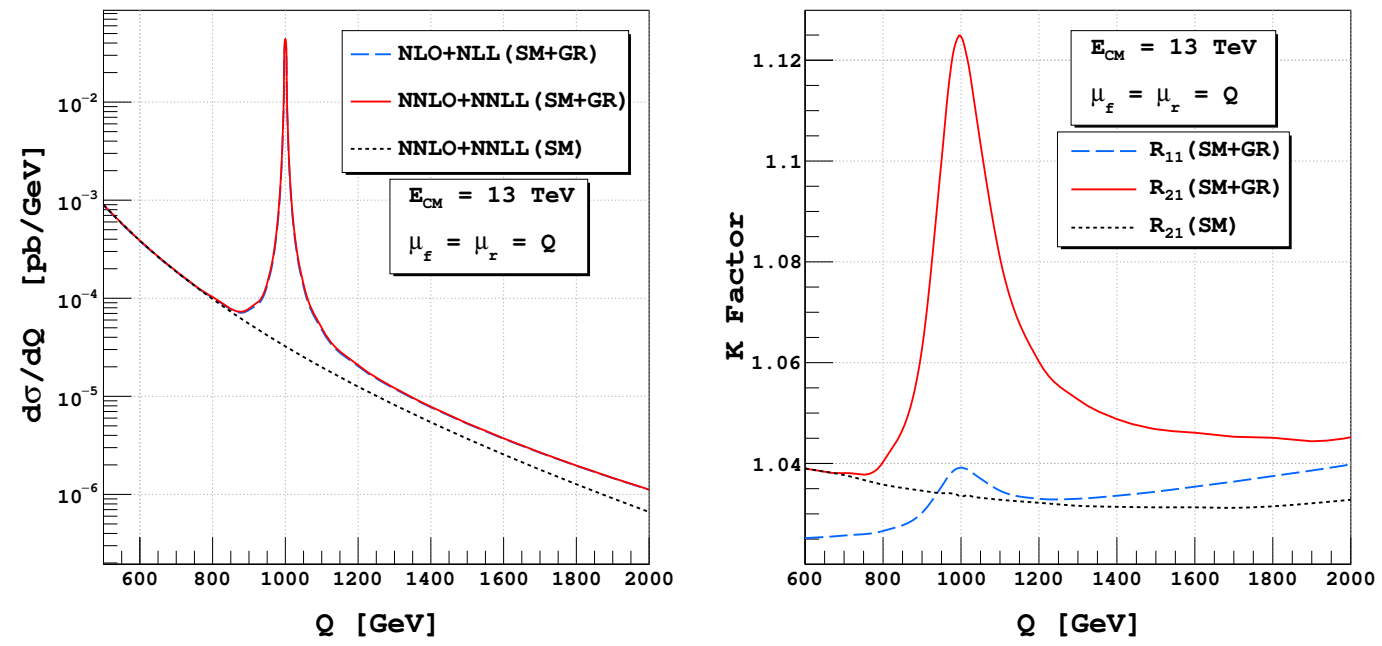

Figure 6. Dilepton invariant mass distributions are presented to NNLO+NNLL QCD for signal (left panel) and the corresponding $K$-factors (right panel).
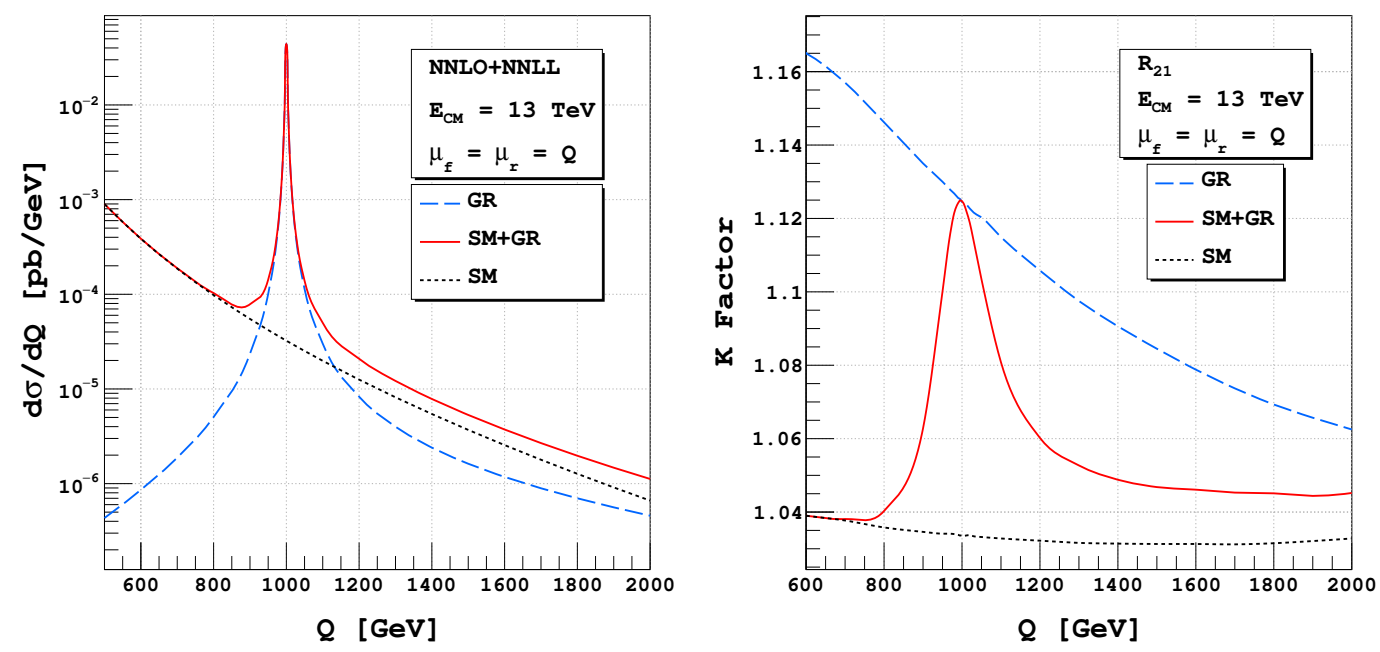

Figure 7. Invariant mass distribution of dilepton for the SM, GR model and for the signal (left) and their corresponding K-factors with respect to NLO (right).

In figure 6, we present the invariant mass distribution to NNLO+NNLL accuracy in the left panel and the corresponding $K$-factors in the right panel for the default choice of model parameters. We observe a significant enhancement in the cross section from lower order to higher order. In figure 7 we present different contributions coming from SM, pure GR and the signal to the invariant mass distributions. We observe that the contribution of gravity to signal is negligible for $Q<900 \mathrm{GeV}$. However, after the resonance the gravity contribution is also prominent which is in contrast to RS scenario as mentioned earlier. In the right panel we present the corresponding $K$-factors for the SM, pure gravity and the signal. For low $Q$ values the signal $K$-factor is equal to that of SM. At the resonance, 


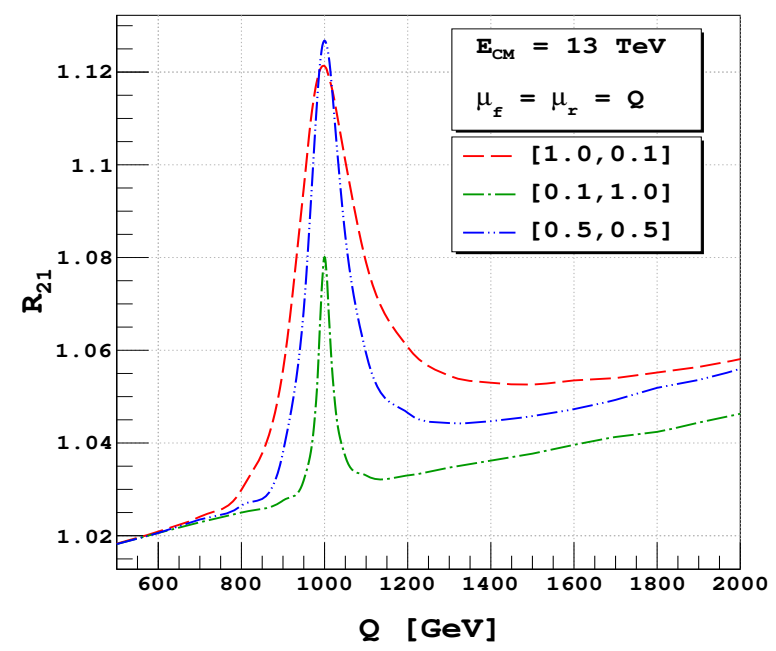

Figure 8. K-factor of signal with respect to NLO at NNLO+NNLL level for different choice of $k_{q}$ and $k_{g}$.

most of the signal contributions are coming from the gravity, therefore, the signal K-factor is almost equal to that of gravity. In figure 8, we show the NNLO+NNLL $K$-factors for different choices of couplings. It can be seen that the higher cross-section is achieved when $k_{g} \lesssim k_{q}$.

We note that these K-factors play an essential role in the experimental searches. In the searches by CMS and ATLAS collaborations for massive gravitons (with universal couplings of $\left.k_{q}=k_{g}=1.0\right)$ at $13 \mathrm{TeV}$ LHC, it is shown in [5, 77] that the inclusion of NLO K-factors $\left(K_{1}\right)$ have tighten the lower limits at $95 \%$ CL on the scale $M_{s}$ of the large extra dimension (ADD) model and on the mass of the first resonance in the warped extra dimension (RS) model. For example, the lower limit on the scale $M_{s}$ in the ADD model is changed from $6.8 \mathrm{TeV}$ to $7.2 \mathrm{TeV}$ in ATLAS data analysis of diphoton events, using a K-factor of 1.4. A similar analysis by CMS collaboration using dilepton events has changed the lower limit on the scale $M_{s}$ from $6.7 \mathrm{TeV}$ to $6.9 \mathrm{TeV}$ when a K-factor of 1.3 is used. Our present analysis involving a generic exotic spin-2 particle estimates even higher order corrections, including resummation effects. These corrections, $R_{21}$ as shown in figure 8 , are found to enhance the cross sections by as large as an additional $10 \%$ and reduce the scale uncertainties by a few percent. These improved theory predictions are thus expected to keep stringent bounds on the model parameters, in the present LHC data analysis as well as that in the future collider experiments of even higher center of mass energies.

We also estimate various theoretical uncertainties in our analysis. We first consider the uncertainties due to the unphysical scales $\mu_{r}$ and $\mu_{f}$. To quantify these uncertainties, we use the canonical 7 -point scale variations by varying $\mu_{r}$ and $\mu_{f}$ simultaneously from $Q / 2$ to $2 Q$ subject to the constraint that the ratio of unphysical scales should not be than 2 and taking the maximum absolute deviations. We put the following constraints on the variation in order to remove extreme combinations,

$$
\left|\ln \frac{\mu_{r}}{Q}\right| \leq \ln 2, \quad\left|\ln \frac{\mu_{f}}{Q}\right| \leq \ln 2, \quad\left|\ln \frac{\mu_{r}}{\mu_{f}}\right| \leq \ln 2
$$



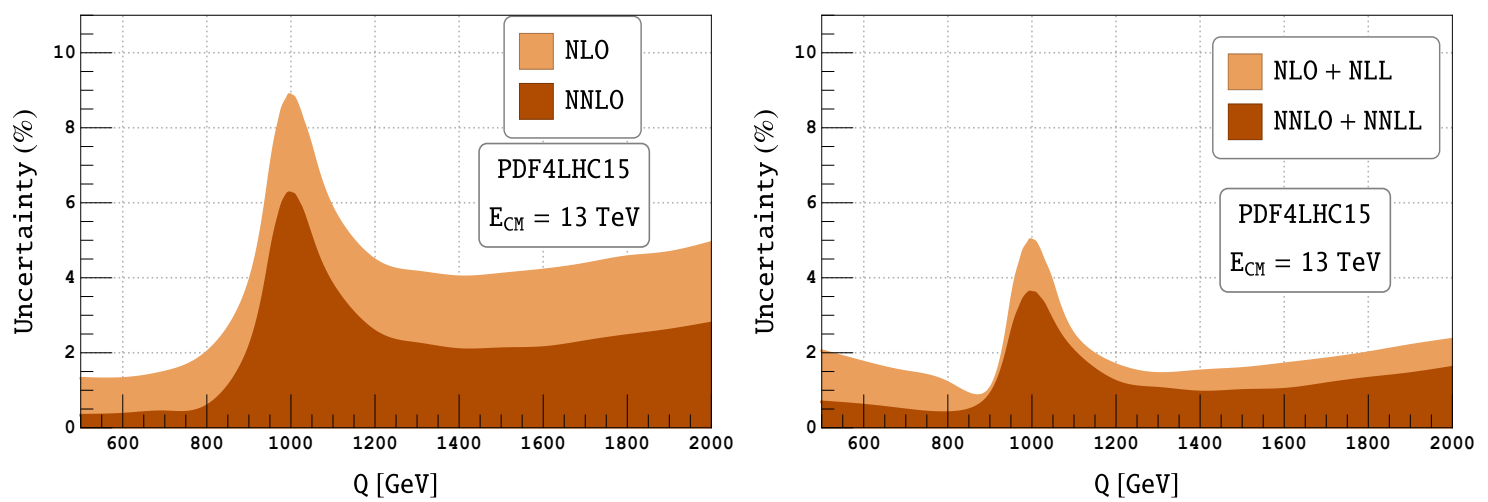

Figure 9. The 7 -point scale variation in the signal in the range $\left(\mu_{r}, \mu_{f}\right) \in(1 / 2,2)$ is shown up to NNLO and NNLO+NNLL for the dilepton invariant mass distribution.

\begin{tabular}{|c|c|c|c|c|c|}
\hline$Q(\mathrm{GeV})$ & 500 & 1000 & 1500 & 2000 & 2500 \\
\hline NNLO & $8.95 \times 10^{-4} \pm 2.0 \%$ & $4.28 \times 10^{-2} \pm 2.2 \%$ & $5.27 \times 10^{-6} \pm 3.4 \%$ & $1.11 \times 10^{-6} \pm 4.2 \%$ & $3.37 \times 10^{-7} \pm 5.1 \%$ \\
\hline NNLO+NNLL & $8.98 \times 10^{-4} \pm 1.9 \%$ & $4.39 \times 10^{-2} \pm 2.2 \%$ & $5.32 \times 10^{-6} \pm 3.3 \%$ & $1.12 \times 10^{-6} \pm 4.2 \%$ & $3.41 \times 10^{-7} \pm 5.0 \%$ \\
\hline
\end{tabular}

Table 1. Intrinsic PDF uncertainties for PDF4LHC15 at $13 \mathrm{TeV}$ LHC.

In figure 9, we present these scale uncertainties both in the fixed order results (left panel) as well as in the resummed results (right panel). At NNLO this uncertainty is estimated to be around $\pm 0.6 \%$ for $Q<M_{G}$, around $\pm 6.3 \%$ at the resonance region $Q=M_{G}=1 \mathrm{TeV}$ and is about $\pm 2.0 \%$ for $Q>M_{G}$. However, the corresponding scale uncertainties for the signal at NNLO+NNLL accuracy found to get significantly reduced, respectively, to around $\pm 0.4 \%, \pm 3.6 \%$ and $\pm 1.0 \%$. We have also estimated the uncertainties only due to the remormalization scale $\mu_{r}$ by varying it from $Q / 2$ to $2 Q$ and keeping $\mu_{f}=Q$ fixed. We observe a significant reduction in the renormalization scale uncertainty from $\pm 1.4 \%$ at NNLO to about $\pm 0.5 \%$ at NNLO+NNLL accuracy. The corresponding factorization scale uncertainties obtained by varying $\mu_{f}$ from $Q / 2$ to $2 Q$ and keeping $\mu_{r}=Q$ fixed are found to get reduced from $5 \%$ at NNLO to $2.6 \%$ at NNLO+NNLL accuracy at the resonance.

Apart from the unphysical scale uncertainties, we also estimate the uncertainties in the fixed order NNLO results as well as in the resummed NNLO+NNLL predictions coming from the non-perturbative PDFs. We have estimated the intrinsic PDF uncertainty for the default choice of PDF4LHC15 PDFs using the recommendation in [78], and present our results in table 1 . We observe that the PDF uncertainty is increasing with invariant mass $(Q)$. However, there is no significant improvement in the PDF uncertainty after inclusion of threshold logarithms. Furthermore, we also compute the cross sections at NNLO+NNLL accuracy for the central set $i=0$ of different PDF groups namely, MMHT2014nnlo68cl [79], CT14nnlo [80], ABMP16_5_nnlo [81] and NNPDF31_nnlo_as_0118 [82] at resonance $Q=$ $M_{G}=1 \mathrm{TeV}$ and at $Q=1500 \mathrm{GeV}$. The corresponding results are found to be different from those of our default PDF choice by as much as $3 \%$.

Although the main focus of our phenomenological study is on the threshold resummation, we have also studied the soft plus virtual (SV) corrections at third order in QCD, $\mathrm{N}^{3} \mathrm{LO}_{\mathrm{SV}}$. We have computed these third order SV coefficients using the three-loop form 


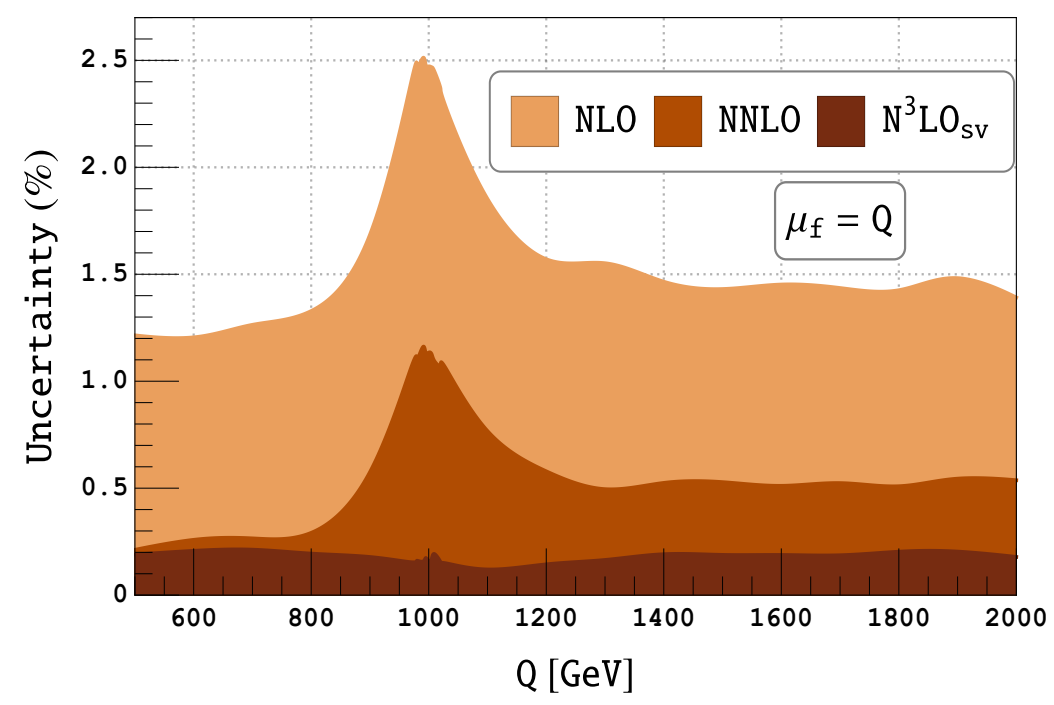

Figure 10. The percentage of uncertainty in the signal due to $\mu_{r}$ scale variation around the central scale $Q$ (in the range $\mu_{r} \in(1 / 2,2) Q$ ) at $\mathrm{N}^{3} \mathrm{LO}$ SV for fixed $\mu_{f}=Q$.

\begin{tabular}{|c|c|c|c|c|c|}
\hline $\mathrm{M}_{\mathrm{G}}(\mathrm{TeV}) \quad \mathrm{E}_{\mathrm{CM}}(\mathrm{TeV})$ & 7 & 8 & 13 & 14 & 28 \\
\hline 1.0 & 1.38 & 1.41 & 1.67 & 1.73 & 3.14 \\
\hline 1.5 & 1.34 & 1.33 & 1.40 & 1.43 & 2.01 \\
\hline 2.0 & 1.39 & 1.35 & 1.33 & 1.34 & 1.63 \\
\hline 2.5 & 1.48 & 1.41 & 1.31 & 1.31 & 1.46 \\
\hline
\end{tabular}

Table 2. K-factor $\left(R_{20}\right)$ for different collider energies at select resonance masses for $\left(k_{q}, k_{g}\right)=$ $(1.0,0.1)$.

factors [48] and exploiting the universality of soft radiations. The new results are presented in appendix C. We finally then give a numerical estimate of these third order corrections by using the running strong coupling constant at 4-loop [83] level. We use the same PDF4LHC15 NNLO set at this order and find that the third order SV result contributes an additional $1 \%$ to the NNLO result at the resonance region. The renormalization scale uncertainty reduces to $\pm 0.2 \%$ at resonance for a canonical variation ${ }^{5}$ within $\mu_{r} \in\{1 / 2,2\} Q$ keeping $\mu_{f}=Q$ fixed. However for a proper estimation of third order QCD corrections (particularly in the region away from threshold region viz. in the lower invariant mass region) one needs to include the regular terms as well as PDF at the third order.

For completeness, we also estimate the size of higher order corrections to NNLO+NNLL for different center of mass energies $\mathrm{E}_{\mathrm{CM}}$ of the incoming protons. In table 2 and table 3 , we present the mass dependent K-factors $R_{20}$ as defined in eq. (3.3) for two different choices of couplings $\left(k_{q}, k_{g}\right)=(1.0,0.1)$ and $(0.1,1.0)$ respectively, for $7,8,13,14$, and $28 \mathrm{TeV}$ hadron colliders. These results show a substantial dependence on the resonance mass as well as

\footnotetext{
${ }^{5}$ Note that it is also possible to estimate theoretical scale uncertainty through a probabilistic description as in $[84,85]$. However we refrain from such a study in this article.
} 


\begin{tabular}{|c|c|c|c|c|c|}
\hline $\mathrm{M}_{\mathrm{G}}(\mathrm{TeV}) \quad \mathrm{E}_{\mathrm{CM}}(\mathrm{TeV})$ & 7 & 8 & 13 & 14 & 28 \\
\hline 1.0 & 1.67 & 1.63 & 1.55 & 1.54 & 1.55 \\
\hline 1.5 & 1.80 & 1.73 & 1.58 & 1.57 & 1.50 \\
\hline 2.0 & 1.98 & 1.87 & 1.63 & 1.61 & 1.50 \\
\hline 2.5 & 2.26 & 2.05 & 1.69 & 1.66 & 1.51 \\
\hline
\end{tabular}

Table 3. K-factor $\left(R_{20}\right)$ for different collider energies at select resonance masses for $\left(k_{q}, k_{g}\right)=$ $(0.1,1.0)$.

on the choice of couplings used. Note that experimental analysis for such models often involves a scaling from LO to take into account the higher order QCD effects at NLO. We stress that in such analysis our mass-dependent K-factors $\left(R_{20}\right)$ could be useful to take into account correctly the QCD effects beyond NLO.

\section{Summary}

We have studied a detailed phenomenology for a generic spin-2 particle production at the $13 \mathrm{TeV}$ LHC. We assumed non-universal coupling of the spin-2 particle with the SM fields. This needs additional UV renormalization for individual operators. After performing UV renormalization of the form factors, we have computed the new SV coefficients at the third order. From these coefficients, we were able to extract the process dependent coefficients needed for threshold resummation. Using the universal threshold exponent, which are already available in the literature, as well as the newly computed process dependent coefficients in this article, we find all the ingredients to perform resummation up to NNLL accuracy. We observe a better perturbative convergence after inclusion of these threshold logarithms. Compared to the fixed order, the cross-section increases by $2.5 \%$ at the NNLL level at the resonance. We show that inclusion of these threshold logarithms are indeed important in taming the theoretical uncertainties to as small as $3.6 \%$ near the resonance. We also discuss the impact of the third order SV coefficients and the corresponding renormalization scale uncertainty. We stress that the $K$ factors in the non-universal case strongly depend on the higher order corrections and a naive scaling from LO will heavily undermine the correct result at $\mathrm{NLO}(\mathrm{NNLO})$ level. Our mass-dependent $K$ factors are thus expected to be useful in the search of such spin-2 resonances at the LHC.

\section{Acknowledgments}

We would like to thank P. Banerjee and P.K. Dhani for useful discussion and providing three loop GR form factor [48] in electronic format. The research of G.D. was supported by the Deutsche Forschungsgemeinschaft (DFG, German Research Foundation) under grant 396021762 - TRR 257 ("Particle Physics Phenomenology after the Higgs Discovery"). G.D. thanks the HoRUS computing facility at Universität Siegen where part of the work has been done. M.C.K. and K.S. would like to thank the high performance computing facility Param Ishan at IIT Guwahati, where a part of the computational work for this study has been carried out. 


\section{A Anomalous dimensions}

The UV anomalous dimensions appearing in eq. (2.10) can be written in terms of renormalized strong coupling as

$$
\gamma_{I J}=\sum_{i=1}^{\infty} a_{s}\left(\mu_{r}\right)^{i} \gamma_{I J}^{(i)}
$$

Up to three-loop the anomalous dimensions are extracted by imposing the universal IR structure of on-shell Form factor in [48] and also collected below for completeness,

$$
\begin{aligned}
\gamma_{G G}^{(1)}= & n_{f}\left(-\frac{2}{3}\right) \\
\gamma_{G G}^{(2)}= & C_{A} n_{f}\left(-\frac{35}{27}\right)+C_{F} n_{f}\left(-\frac{74}{27}\right) \\
\gamma_{G G}^{(3)}= & C_{A}^{2} n_{f}\left(-\frac{3589}{162}+24 \zeta_{3}\right)+C_{A} C_{F} n_{f}\left(\frac{139}{9}-\frac{104}{3} \zeta_{3}\right)+C_{F}^{2} n_{f}\left(-\frac{2155}{243}+\frac{32}{3} \zeta_{3}\right) \\
& +C_{A} n_{f}^{2}\left(\frac{1058}{243}\right)+C_{F} n_{f}^{2}\left(-\frac{173}{243}\right) \\
\gamma_{Q Q}^{(1)}= & C_{F}\left(-\frac{8}{3}\right) \\
\gamma_{Q Q}^{(2)}= & C_{A} C_{F}\left(-\frac{376}{27}\right)+C_{F}^{2}\left(\frac{112}{27}\right)+C_{F} n_{f}\left(\frac{104}{27}\right), \\
\gamma_{Q Q}^{(3)}= & C_{A}^{2} C_{F}\left(-\frac{20920}{243}-\frac{64}{3} \zeta_{3}\right)+C_{A} C_{F}^{2}\left(\frac{8528}{243}+64 \zeta_{3}\right)+C_{F}^{3}\left(\frac{560}{243}-\frac{128}{3} \zeta_{3}\right) \\
& +C_{A} C_{F} n_{f}\left(\frac{22}{9}+\frac{128}{3} \zeta_{3}\right)+C_{F}^{2} n_{f}\left(\frac{7094}{243}-\frac{128}{3} \zeta_{3}\right)+C_{F} n_{f}^{2}\left(\frac{284}{81}\right) .
\end{aligned}
$$

Note that the conservation of the sum of the operators fixes the remaining anomalous dimensions i.e.

$$
\begin{aligned}
\gamma_{G G}+\gamma_{Q G} & =0, \\
\gamma_{G Q}+\gamma_{Q Q} & =0 .
\end{aligned}
$$

\section{B Process dependent resum coefficients}

The process-dependent resum coefficients are collected here for both quark and gluon channels with exact dependence of renormalization and factorization scales. Notice that the coefficients now explicitly depend on the gluon and quark coupling to spin-2 particle through the ratio $r_{q}=k_{g} / k_{q}$ for quark initiated subprocess and through $r_{g}=k_{q} / k_{g}$ for gluon initiated subprocess. At each order the coefficients take the following form,

$$
g_{0, p}^{(i), I}=g_{00, p}^{(i), I}+r_{p} g_{01, p}^{(i), I}+r_{p}^{2} g_{02, p}^{(i), I} .
$$

Notice that the universal case is recovered after realising $r_{p}=1$. Defining $L_{q r}=\ln \left(Q^{2} / \mu_{r}^{2}\right)$ and $L_{f r}=\ln \left(\mu_{f}^{2} / \mu_{r}^{2}\right)$,

$$
\begin{aligned}
g_{0, g}^{(1), \mathrm{GR}}= & r_{g}\left\{\left(\frac{35}{9}+\left(-\frac{4}{3}\right) L_{q r}\right) n_{f}\right\}+\left\{\left(-\frac{203}{9}+16 \zeta_{2}+\left(-\frac{22}{3}\right) L_{f r}+\left(\frac{22}{3}\right) L_{q r}\right) C_{A}\right. \\
& \left.+\left(\left(\frac{4}{3}\right) L_{f r}\right) n_{f}\right\},
\end{aligned}
$$




$$
\begin{aligned}
g_{0, g}^{(2), \mathrm{GR}}= & r_{g}\left\{\left(-\frac{10295}{162}+16 \zeta_{3}+\frac{442}{9} \zeta_{2}+\left(-\frac{770}{27}\right) L_{f r}+\left(-\frac{22}{3}\right) L_{q r}^{2}+\left(\frac{88}{9}\right) L_{q r} L_{f r}\right.\right. \\
& \left.+\left(\frac{1127}{27}-\frac{64}{3} \zeta_{2}\right) L_{q r}\right) C_{A} n_{f}+\left(\frac{598}{81}-16 \zeta_{3}+\frac{128}{9} \zeta_{2}+\left(-\frac{16}{9}\right) L_{q r}^{2}\right. \\
& \left.\left.+\left(\frac{44}{9}\right) L_{q r}\right) C_{F} n_{f}+\left(\left(-\frac{16}{9}\right) L_{q r} L_{f r}+\left(\frac{140}{27}\right) L_{f r}\right) n_{f}^{2}\right\}+r_{g}^{2}\left\{\left(\frac{1225}{324}+\frac{8}{3} \zeta_{2}+(\right.\right. \\
& \left.\left.\left.-\frac{70}{27}\right) L_{q r}+\left(\frac{4}{9}\right) L_{q r}^{2}\right) n_{f}^{2}\right\}+\left\{\left(\frac{1049}{81}-\frac{128}{9} \zeta_{2}+\left(-\frac{80}{9}\right) L_{q r}+\left(\frac{16}{9}\right) L_{q r}^{2}\right.\right. \\
& \left.+(4) L_{f r}\right) C_{F} n_{f}+\left(\frac{7801}{324}+\frac{88}{9} \zeta_{3}-\frac{1312}{9} \zeta_{2}+92 \zeta_{2}^{2}+\left(-\frac{1657}{27}+24 \zeta_{3}+\frac{176}{3} \zeta_{2}\right) L_{q r}\right. \\
& \left.+\left(-\frac{484}{9}\right) L_{q r} L_{f r}+\left(\frac{121}{9}\right) L_{q r}^{2}+\left(\frac{121}{3}\right) L_{f r}^{2}+\left(\frac{3890}{27}-24 \zeta_{3}-\frac{352}{3} \zeta_{2}\right) L_{f r}\right) C_{A}^{2} \\
& +\left(\frac{3656}{81}-\frac{16}{9} \zeta_{3}-\frac{112}{3} \zeta_{2}+\left(-\frac{668}{27}+\frac{64}{3} \zeta_{2}\right) L_{f r}+\left(-\frac{160}{9}+\frac{32}{3} \zeta_{2}\right) L_{q r}+(\mathrm{B} .3)\right. \\
& \left.\left.\left.-\frac{44}{3}\right) L_{f r}^{2}+\left(\frac{22}{9}\right) L_{q r}^{2}+\left(\frac{88}{9}\right) L_{q r} L_{f r}\right) C_{A} n_{f}+\left(\left(\frac{4}{3}\right) L_{f r}^{2}\right) n_{f}^{2}\right\},
\end{aligned}
$$

$g_{0, g}^{(3), \mathrm{GR}}=r_{g}\left\{\left(-\frac{464173}{3645}+80 \zeta_{5}-\frac{256}{45} \zeta_{3}+\frac{14708}{81} \zeta_{2}-\frac{704}{3} \zeta_{2} \zeta_{3}+\frac{3376}{15} \zeta_{2}^{2}+\left(-\frac{13156}{243}+\frac{352}{3} \zeta_{3}\right.\right.\right.$

$\left.-\frac{2816}{27} \zeta_{2}\right) L_{f r}+\left(-\frac{968}{27}\right) L_{q r} L_{f r}+\left(-\frac{176}{27}\right) L_{q r}^{3}+\left(\frac{352}{27}\right) L_{q r}^{2} L_{f r}+\left(\frac{2128}{81}\right.$

$\left.\left.-\frac{256}{9} \zeta_{2}\right) L_{q r}^{2}+\left(\frac{9530}{243}-\frac{80}{3} \zeta_{3}+\frac{1168}{27} \zeta_{2}\right) L_{q r}\right) C_{A} C_{F} n_{f}+\left(-\frac{2410567}{21870}-\frac{760}{3} \zeta_{5}\right.$

$+\frac{50009}{405} \zeta_{3}-\frac{18251}{81} \zeta_{2}+\frac{716}{3} \zeta_{2} \zeta_{3}+\frac{6449}{45} \zeta_{2}^{2}+\left(-\frac{22490}{81}+32 \zeta_{3}+\frac{1408}{9} \zeta_{2}\right) L_{q r} L_{f r}$

$+\left(-\frac{484}{9}\right) L_{q r} L_{f r}^{2}+\left(-\frac{484}{81}\right) L_{q r}^{3}+\left(\frac{5092}{243}+\frac{3464}{27} \zeta_{3}+\frac{1376}{9} \zeta_{2}-\frac{368}{3} \zeta_{2}^{2}\right) L_{q r}$

$+\left(\frac{668}{27}-32 \zeta_{3}-\frac{352}{9} \zeta_{2}\right) L_{q r}^{2}+\left(\frac{484}{9}\right) L_{q r}^{2} L_{f r}+\left(\frac{4235}{27}\right) L_{f r}^{2}+\left(\frac{93085}{243}-\frac{632}{3} \zeta_{3}\right.$

$\left.\left.-\frac{9724}{27} \zeta_{2}\right) L_{f r}\right) C_{A}^{2} n_{f}+\left(-\frac{35176}{3645}+160 \zeta_{5}-\frac{29368}{405} \zeta_{3}-\frac{6016}{81} \zeta_{2}-\frac{64}{15} \zeta_{2}^{2}+\left(-\frac{3446}{243}\right.\right.$

$\left.\left.-\frac{64}{3} \zeta_{3}+\frac{1024}{27} \zeta_{2}\right) L_{q r}+\left(-\frac{128}{81}\right) L_{q r}^{3}+\left(\frac{752}{81}\right) L_{q r}^{2}\right) C_{F}^{2} n_{f}+\left(\frac{16087}{486}+\frac{3296}{81} \zeta_{3}\right.$

$-\frac{4216}{81} \zeta_{2}+\left(-\frac{11186}{243}-\frac{32}{3} \zeta_{3}+\frac{128}{9} \zeta_{2}\right) L_{q r}+\left(-\frac{64}{27}\right) L_{q r}^{2} L_{f r}+\left(-\frac{160}{81}\right) L_{q r}^{3}$

$\left.+\left(\frac{32}{27}\right) L_{q r} L_{f r}+\left(\frac{1340}{81}\right) L_{q r}^{2}+\left(\frac{6172}{243}-\frac{64}{3} \zeta_{3}+\frac{512}{27} \zeta_{2}\right) L_{f r}\right) C_{F} n_{f}^{2}+\left(\frac{1404698}{10935}\right.$

$-\frac{7384}{135} \zeta_{3}-\frac{9112}{81} \zeta_{2}-\frac{32}{45} \zeta_{2}^{2}+\left(-\frac{23468}{243}+\frac{352}{27} \zeta_{3}+\frac{2228}{27} \zeta_{2}\right) L_{q r}+\left(-\frac{15550}{243}+\frac{64}{3} \zeta_{3}\right.$

$\left.+\frac{1768}{27} \zeta_{2}\right) L_{f r}+\left(-\frac{1540}{27}\right) L_{f r}^{2}+\left(-\frac{88}{9}\right) L_{q r}^{2} L_{f r}+\left(-\frac{220}{81}\right) L_{q r}^{3}+\left(\frac{176}{9}\right) L_{q r} L_{f r}^{2}$

$\left.+\left(\frac{2125}{81}-\frac{128}{9} \zeta_{2}\right) L_{q r}^{2}+\left(\frac{3932}{81}-\frac{256}{9} \zeta_{2}\right) L_{q r} L_{f r}\right) C_{A} n_{f}^{2}+\left(\left(-\frac{16}{9}\right) L_{q r} L_{f r}^{2}\right.$ 


$$
\begin{aligned}
& \left.\left.+\left(\frac{140}{27}\right) L_{f r}^{2}\right) n_{f}^{3}\right\}+r_{g}^{2}\left\{\left(-\frac{55825}{1458}+\frac{280}{9} \zeta_{3}+\frac{1141}{27} \zeta_{2}+\frac{128}{3} \zeta_{2}^{2}+\left(-\frac{13475}{486}\right.\right.\right. \\
& \left.-\frac{176}{9} \zeta_{2}\right) L_{f r}+\left(-\frac{1057}{81}+\frac{64}{9} \zeta_{2}\right) L_{q r}^{2}+\left(-\frac{88}{27}\right) L_{q r}^{2} L_{f r}+\left(\frac{44}{27}\right) L_{q r}^{3} \\
& \left.+\left(\frac{1540}{81}\right) L_{q r} L_{f r}+\left(\frac{9070}{243}-\frac{32}{3} \zeta_{3}-\frac{884}{27} \zeta_{2}\right) L_{q r}\right) C_{A} n_{f}^{2}+\left(\frac{10465}{729}-\frac{280}{9} \zeta_{3}+\frac{656}{81} \zeta_{2}\right. \\
& \left.+\left(-\frac{544}{81}\right) L_{q r}^{2}+\left(\frac{32}{27}\right) L_{q r}^{3}+\left(\frac{1114}{243}+\frac{32}{3} \zeta_{3}+\frac{128}{27} \zeta_{2}\right) L_{q r}\right) C_{F} n_{f}^{2}+(( \\
& \left.\left.\left.-\frac{280}{81}\right) L_{q r} L_{f r}+\left(\frac{16}{27}\right) L_{q r}^{2} L_{f r}+\left(\frac{1225}{243}+\frac{32}{9} \zeta_{2}\right) L_{f r}\right) n_{f}^{3}\right\}+\left\{\left(-\frac{303707}{810}\right.\right. \\
& +\frac{5156}{9} \zeta_{5}-\frac{81074}{135} \zeta_{3}+96 \zeta_{3}^{2}-\frac{697}{9} \zeta_{2}+48 \zeta_{2} \zeta_{3}+\frac{6248}{27} \zeta_{2}^{2}+\frac{3872}{15} \zeta_{2}^{3}+\left(-\frac{17105}{27}\right. \\
& \left.+264 \zeta_{3}+\frac{1936}{3} \zeta_{2}\right) L_{f r}^{2}+\left(-\frac{5324}{27}\right) L_{f r}^{3}+\left(-\frac{1319}{9}-160 \zeta_{5}-184 \zeta_{3}+\frac{496}{3} \zeta_{2}\right. \\
& \left.+352 \zeta_{2} \zeta_{3}+\frac{44}{3} \zeta_{2}^{2}\right) L_{q r}+\left(-\frac{2662}{27}\right) L_{q r}^{2} L_{f r}+\left(\frac{110}{3}+88 \zeta_{3}\right) L_{q r}^{2}+\left(\frac{109651}{486}+160 \zeta_{5}\right. \\
& \left.+\frac{3032}{27} \zeta_{3}+\frac{19504}{27} \zeta_{2}-352 \zeta_{2} \zeta_{3}-\frac{2068}{3} \zeta_{2}^{2}\right) L_{f r}+\left(\frac{23782}{81}-352 \zeta_{3}-\frac{3872}{9} \zeta_{2}\right) L_{q r} L_{f r} \\
& \left.+\left(\frac{2662}{9}\right) L_{q r} L_{f r}^{2}\right) C_{A}^{3}+\left(-\frac{442583}{4374}+\frac{16}{9} \zeta_{3}+\frac{9196}{81} \zeta_{2}-\frac{688}{45} \zeta_{2}^{2}+\left(-\frac{640}{27}\right.\right. \\
& \left.+\frac{128}{9} \zeta_{2}\right) L_{q r} L_{f r}+\left(-\frac{176}{9}\right) L_{f r}^{3}+\left(-\frac{464}{27}+\frac{64}{3} \zeta_{2}\right) L_{f r}^{2}+\left(-\frac{320}{27}+\frac{64}{9} \zeta_{2}\right) L_{q r}^{2} \\
& +\left(\frac{88}{81}\right) L_{q r}^{3}+\left(\frac{88}{27}\right) L_{q r}^{2} L_{f r}+\left(\frac{88}{9}\right) L_{q r} L_{f r}^{2}+\left(\frac{13291}{243}-\frac{64}{27} \zeta_{3}-\frac{448}{9} \zeta_{2}\right) L_{q r} \\
& \left.+\left(\frac{13841}{243}-\frac{64}{27} \zeta_{3}-\frac{448}{9} \zeta_{2}\right) L_{f r}\right) C_{A} n_{f}^{2}+\left(-\frac{46372}{729}-\frac{128}{81} \zeta_{3}+\frac{5504}{81} \zeta_{2}+(\right. \\
& \left.-\frac{320}{27}\right) L_{q r} L_{f r}+\left(-\frac{688}{81}\right) L_{q r}^{2}+\left(\frac{64}{81}\right) L_{q r}^{3}+\left(\frac{64}{27}\right) L_{q r}^{2} L_{f r}+\left(\frac{28}{3}\right) L_{f r}^{2}+\left(\frac{3602}{243}\right. \\
& \left.\left.-\frac{512}{27} \zeta_{2}\right) L_{f r}+\left(\frac{8776}{243}-\frac{512}{27} \zeta_{2}\right) L_{q r}\right) C_{F} n_{f}^{2}+\left(-\frac{62429}{3645}-\frac{10592}{405} \zeta_{3}+\frac{6016}{81} \zeta_{2}\right. \\
& +\frac{64}{15} \zeta_{2}^{2}+\left(-\frac{752}{81}\right) L_{q r}^{2}+\left(\frac{128}{81}\right) L_{q r}^{3}+\left(\frac{3932}{243}+\frac{64}{3} \zeta_{3}-\frac{1024}{27} \zeta_{2}\right) L_{q r} \\
& \left.+(-2) L_{f r}\right) C_{F}^{2} n_{f}+\left(\frac{5410051}{21870}-\frac{1112}{9} \zeta_{5}+\frac{95998}{405} \zeta_{3}+\frac{8804}{81} \zeta_{2}-144 \zeta_{2} \zeta_{3}\right. \\
& -\frac{42028}{135} \zeta_{2}^{2}+\left(-\frac{95572}{243}+\frac{2144}{27} \zeta_{3}+\frac{4592}{27} \zeta_{2}+\frac{376}{3} \zeta_{2}^{2}\right) L_{f r}+\left(-\frac{968}{9}\right) L_{q r} L_{f r}^{2}+( \\
& \left.-\frac{1046}{27}+16 \zeta_{3}+\frac{352}{9} \zeta_{2}\right) L_{q r}^{2}+\left(\frac{484}{81}\right) L_{q r}^{3}+\left(\frac{9029}{243}-\frac{2384}{27} \zeta_{3}-\frac{1712}{9} \zeta_{2}+120 \zeta_{2}^{2}\right) L_{q r} \\
& \left.+\left(\frac{7100}{81}+32 \zeta_{3}\right) L_{q r} L_{f r}+\left(\frac{968}{9}\right) L_{f r}^{3}+\left(\frac{5662}{27}-48 \zeta_{3}-\frac{704}{3} \zeta_{2}\right) L_{f r}^{2}\right) C_{A}^{2} n_{f} \\
& +\left(\frac{1056967}{3645}-\frac{6256}{45} \zeta_{3}-\frac{12152}{81} \zeta_{2}+128 \zeta_{2} \zeta_{3}-\frac{2032}{9} \zeta_{2}^{2}+\left(-\frac{38495}{243}+\frac{4544}{27} \zeta_{2}\right) L_{f r}\right.
\end{aligned}
$$




$$
\begin{aligned}
& +\left(-\frac{154}{3}\right) L_{f r}^{2}+\left(-\frac{2722}{81}+\frac{256}{9} \zeta_{2}\right) L_{q r}^{2}+\left(-\frac{5075}{243}+\frac{80}{3} \zeta_{3}-\frac{2032}{27} \zeta_{2}\right) L_{q r}+( \\
& \left.\left.\left.-\frac{352}{27}\right) L_{q r}^{2} L_{f r}+\left(\frac{176}{27}\right) L_{q r}^{3}+\left(\frac{2552}{27}\right) L_{q r} L_{f r}\right) C_{A} C_{F} n_{f}+\left(\left(\frac{32}{27}\right) L_{f r}^{3}\right) n_{f}^{3}\right\}
\end{aligned}
$$

$$
\begin{aligned}
g_{0, q}^{(1), \mathrm{GR}}= & r_{q}\left\{\left(\frac{68}{9}+\left(-\frac{16}{3}\right) L_{q r}\right) C_{F}\right\}+\left\{\left(-\frac{248}{9}+16 \zeta_{2}+\left(\frac{34}{3}\right) L_{q r}+(-6) L_{f r}\right) C_{F}\right\}, \\
g_{0, q}^{(2), \mathrm{GR}}= & r_{q}\left\{\left(-\frac{17998}{81}+\frac{832}{9} \zeta_{2}+\left(-\frac{160}{3}\right) L_{q r}^{2}+\left(-\frac{136}{3}\right) L_{f r}+\left(\frac{5960}{27}-\frac{256}{3} \zeta_{2}\right) L_{q r}\right.\right. \\
& \left.+(32) L_{q r} L_{f r}\right) C_{F}^{2}+\left(-\frac{2332}{81}+\frac{64}{3} \zeta_{2}+\left(-\frac{32}{9}\right) L_{q r}^{2}+\left(\frac{160}{9}\right) L_{q r}\right) C_{F} n_{f}+\left(\frac{7826}{81}\right. \\
& \left.\left.-\frac{472}{9} \zeta_{2}+\left(-\frac{500}{9}\right) L_{q r}+\left(\frac{88}{9}\right) L_{q r}^{2}\right) C_{A} C_{F}\right\}+r_{q}^{2}\left\{\left(\frac{1156}{81}+\frac{128}{3} \zeta_{2}+\left(-\frac{544}{27}\right) L_{q r}\right.\right. \\
& \left.\left.+\left(\frac{64}{9}\right) L_{q r}^{2}\right) C_{F}^{2}\right\}+\left\{\left(-\frac{84773}{324}+\frac{1180}{9} \zeta_{3}+\frac{1336}{9} \zeta_{2}-\frac{92}{5} \zeta_{2}^{2}+\left(-\frac{187}{9}\right) L_{q r}^{2}+(\right.\right. \\
& \left.\left.-\frac{17}{3}+24 \zeta_{3}-\frac{88}{3} \zeta_{2}\right) L_{f r}+\left(\frac{1211}{9}-24 \zeta_{3}-\frac{88}{3} \zeta_{2}\right) L_{q r}+(11) L_{f r}^{2}\right) C_{A} C_{F}+\left(\frac{8813}{162}\right. \\
& +\frac{8}{9} \zeta_{3}-\frac{112}{3} \zeta_{2}+\left(-\frac{286}{9}+\frac{16}{3} \zeta_{2}\right) L_{q r}+\left(\frac{2}{3}+\frac{16}{3} \zeta_{2}\right) L_{f r}+\left(\frac{50}{9}\right) L_{q r}^{2} \\
& \left.+(-2) L_{f r}^{2}\right) C_{F} n_{f}+\left(\frac{43093}{108}-124 \zeta_{3}-\frac{3286}{9} \zeta_{2}+\frac{552}{5} \zeta_{2}^{2}+\left(-\frac{8575}{27}+48 \zeta_{3}\right.\right. \\
& \left.+\frac{472}{3} \zeta_{2}\right) L_{q r}+\left(\frac{578}{9}\right) L_{q r}^{2}+\left(\frac{487}{3}-48 \zeta_{3}-72 \zeta_{2}\right) L_{f r}+(-68) L_{q r} L_{f r} \\
& \left.\left.+(18) L_{f r}^{2}\right) C_{F}^{2}\right\}, \\
& (\mathrm{B} .6)
\end{aligned}
$$

$g_{0, q}^{(3), \mathrm{GR}}=r_{q}\left\{\left(-\frac{10514857}{2187}+\frac{1280}{3} \zeta_{5}+\frac{44288}{81} \zeta_{3}+\frac{270848}{81} \zeta_{2}+\frac{256}{3} \zeta_{2} \zeta_{3}-\frac{15536}{15} \zeta_{2}^{2}+(\right.\right.$

$\left.-\frac{131140}{81}+128 \zeta_{3}+\frac{2816}{9} \zeta_{2}\right) L_{q r}^{2}+\left(-\frac{16808}{27}+\frac{544}{3} \zeta_{3}+\frac{2512}{27} \zeta_{2}\right) L_{f r}+($

$\left.-\frac{176}{3}\right) L_{q r} L_{f r}^{2}+\left(-\frac{176}{3}\right) L_{q r}^{2} L_{f r}+\left(\frac{748}{9}\right) L_{f r}^{2}+\left(\frac{1760}{9}\right) L_{q r}^{3}+\left(\frac{3272}{9}-128 \zeta_{3}\right.$

$\left.\left.+\frac{1408}{9} \zeta_{2}\right) L_{q r} L_{f r}+\left(\frac{1178048}{243}-\frac{20320}{27} \zeta_{3}-\frac{58000}{27} \zeta_{2}+\frac{1472}{15} \zeta_{2}^{2}\right) L_{q r}\right) C_{A} C_{F}^{2}+($

$-\frac{1268944}{2187}-\frac{10528}{81} \zeta_{3}+\frac{60512}{81} \zeta_{2}-\frac{896}{45} \zeta_{2}^{2}+\left(-\frac{10780}{81}\right) L_{q r}^{2}+\left(\frac{1232}{81}\right) L_{q r}^{3}$

$\left.+\left(\frac{107948}{243}+\frac{256}{3} \zeta_{3}-\frac{784}{3} \zeta_{2}\right) L_{q r}\right) C_{A} C_{F} n_{f}+\left(\frac{39772}{729}-\frac{320}{3} \zeta_{2}+\left(-\frac{4096}{81}\right.\right.$

$\left.\left.+\frac{128}{3} \zeta_{2}\right) L_{q r}+\left(-\frac{64}{27}\right) L_{q r}^{3}+\left(\frac{160}{9}\right) L_{q r}^{2}\right) C_{F} n_{f}^{2}+\left(\frac{2196934}{2187}+160 \zeta_{3}-\frac{77536}{81} \zeta_{2}\right.$

$+\frac{1792}{5} \zeta_{2}^{2}+\left(-\frac{280736}{243}-\frac{2432}{27} \zeta_{3}+\frac{16064}{27} \zeta_{2}\right) L_{q r}+\left(-\frac{992}{9}-\frac{256}{9} \zeta_{2}\right) L_{q r} L_{f r}+($ 


$$
\begin{aligned}
& \left.-\frac{4768}{81}\right) L_{q r}^{3}+\left(-\frac{136}{9}\right) L_{f r}^{2}+\left(\frac{32}{3}\right) L_{q r} L_{f r}^{2}+\left(\frac{64}{3}\right) L_{q r}^{2} L_{f r}+\left(\frac{1600}{9}\right. \\
& \left.\left.-\frac{2368}{27} \zeta_{2}\right) L_{f r}+\left(\frac{35560}{81}-\frac{256}{3} \zeta_{2}\right) L_{q r}^{2}\right) C_{F}^{2} n_{f}+\left(\frac{3381256}{2187}-480 \zeta_{5}+\frac{29972}{81} \zeta_{3}\right. \\
& -\frac{3344}{3} \zeta_{2}-\frac{208}{3} \zeta_{2} \zeta_{3}+\frac{2324}{45} \zeta_{2}^{2}+\left(-\frac{234820}{243}-\frac{128}{3} \zeta_{3}+\frac{10384}{27} \zeta_{2}\right) L_{q r}+( \\
& \left.\left.-\frac{1936}{81}\right) L_{q r}^{3}+\left(\frac{6316}{27}\right) L_{q r}^{2}\right) C_{A}^{2} C_{F}+\left(\frac{263089}{81}-\frac{72176}{81} \zeta_{3}-\frac{6184}{3} \zeta_{2}+\frac{1696}{5} \zeta_{2}^{2}+(\right. \\
& \left.-\frac{1041604}{243}+\frac{2816}{3} \zeta_{3}+\frac{72896}{27} \zeta_{2}-\frac{2944}{5} \zeta_{2}^{2}\right) L_{q r}+\left(-\frac{11776}{9}+256 \zeta_{3}\right. \\
& \left.+384 \zeta_{2}\right) L_{q r} L_{f r}+\left(-\frac{21728}{81}\right) L_{q r}^{3}+\left(\frac{35384}{27}-\frac{1088}{3} \zeta_{3}-\frac{1120}{3} \zeta_{2}\right) L_{f r}+\left(\frac{150136}{81}\right. \\
& \left.\left.\left.-256 \zeta_{3}-\frac{2176}{3} \zeta_{2}\right) L_{q r}^{2}+(-96) L_{q r} L_{f r}^{2}+(136) L_{f r}^{2}+(320) L_{q r}^{2} L_{f r}\right) C_{F}^{3}\right\} \\
& +r_{q}^{2}\left\{\left(-\frac{325244}{729}-\frac{23360}{27} \zeta_{2}+\frac{2048}{3} \zeta_{2}^{2}+\left(-\frac{29632}{81}+\frac{1024}{9} \zeta_{2}\right) L_{q r}^{2}+\left(-\frac{2312}{27}\right.\right.\right. \\
& \left.-256 \zeta_{2}\right) L_{f r}+\left(-\frac{128}{3}\right) L_{q r}^{2} L_{f r}+\left(\frac{1664}{27}\right) L_{q r}^{3}+\left(\frac{1088}{9}\right) L_{q r} L_{f r}+\left(\frac{172408}{243}\right. \\
& \left.\left.+\frac{256}{27} \zeta_{2}\right) L_{q r}\right) C_{F}^{3}+\left(-\frac{79288}{729}-\frac{5504}{27} \zeta_{2}+\left(-\frac{4928}{81}\right) L_{q r}^{2}+\left(\frac{256}{27}\right) L_{q r}^{3}+\left(\frac{34976}{243}\right.\right. \\
& \left.\left.+\frac{512}{9} \zeta_{2}\right) L_{q r}\right) C_{F}^{2} n_{f}+\left(\frac{266084}{729}+\frac{55952}{81} \zeta_{2}+\left(-\frac{113608}{243}-\frac{4672}{27} \zeta_{2}\right) L_{q r}+(\right. \\
& \left.\left.\left.-\frac{704}{27}\right) L_{q r}^{3}+\left(\frac{14992}{81}\right) L_{q r}^{2}\right) C_{A} C_{F}^{2}\right\}+\left\{\left(-\frac{6131417}{1458}+\frac{3344}{3} \zeta_{5}+\frac{188276}{81} \zeta_{3}+32 \zeta_{3}^{2}\right.\right. \\
& +\frac{110552}{27} \zeta_{2}-1424 \zeta_{2} \zeta_{3}-\frac{34492}{15} \zeta_{2}^{2}+\frac{169504}{315} \zeta_{2}^{3}+\left(-\frac{42127}{18}+480 \zeta_{5}+\frac{5792}{3} \zeta_{3}\right. \\
& \left.+\frac{4336}{3} \zeta_{2}-704 \zeta_{2} \zeta_{3}-\frac{1968}{5} \zeta_{2}^{2}\right) L_{f r}+\left(-\frac{49402}{27}+544 \zeta_{3}+\frac{6800}{9} \zeta_{2}\right) L_{q r}^{2}+(-478 \\
& \left.+288 \zeta_{3}+144 \zeta_{2}\right) L_{f r}^{2}+\left(-\frac{1156}{3}\right) L_{q r}^{2} L_{f r}+\left(\frac{19652}{81}\right) L_{q r}^{3}+\left(\frac{16844}{9}-832 \zeta_{3}\right. \\
& \left.-672 \zeta_{2}\right) L_{q r} L_{f r}+\left(\frac{760175}{162}-480 \zeta_{5}-\frac{7520}{3} \zeta_{3}-\frac{10576}{3} \zeta_{2}+704 \zeta_{2} \zeta_{3}+\frac{4912}{5} \zeta_{2}^{2}\right) L_{q r} \\
& \left.+(-36) L_{f r}^{3}+(204) L_{q r} L_{f r}^{2}\right) C_{F}^{3}+\left(-\frac{31255393}{8748}-\frac{2852}{3} \zeta_{5}+\frac{309353}{81} \zeta_{3}-\frac{400}{3} \zeta_{3}^{2}\right. \\
& +\frac{181355}{81} \zeta_{2}-\frac{7036}{9} \zeta_{2} \zeta_{3}-\frac{3529}{27} \zeta_{2}^{2}+\frac{7088}{63} \zeta_{2}^{3}+\left(-\frac{15055}{27}+88 \zeta_{3}+\frac{968}{9} \zeta_{2}\right) L_{q r}^{2}+( \\
& \left.-\frac{242}{9}\right) L_{f r}^{3}+\left(\frac{4114}{81}\right) L_{q r}^{3}+\left(\frac{493}{9}-88 \zeta_{3}+\frac{968}{9} \zeta_{2}\right) L_{f r}^{2}+\left(\frac{1657}{18}-80 \zeta_{5}+\frac{3104}{9} \zeta_{3}\right. \\
& \left.\left.-\frac{8992}{27} \zeta_{2}+4 \zeta_{2}^{2}\right) L_{f r}+\left(\frac{561610}{243}+80 \zeta_{5}-\frac{34120}{27} \zeta_{3}-\frac{8432}{9} \zeta_{2}+\frac{1964}{15} \zeta_{2}^{2}\right) L_{q r}\right) C_{A}^{2} C_{F} \\
& +\left(-\frac{2467183}{2187}-\frac{608}{9} \zeta_{5}+\frac{13960}{27} \zeta_{3}+\frac{115336}{81} \zeta_{2}-\frac{256}{3} \zeta_{2} \zeta_{3}-\frac{75568}{135} \zeta_{2}^{2}+\left(-\frac{40028}{81}\right.\right.
\end{aligned}
$$




$$
\begin{aligned}
& \left.+32 \zeta_{3}+\frac{400}{3} \zeta_{2}\right) L_{q r}^{2}+\left(-\frac{2689}{9}+\frac{256}{3} \zeta_{3}+\frac{2008}{27} \zeta_{2}+\frac{272}{5} \zeta_{2}^{2}\right) L_{f r}+\left(-\frac{100}{3}\right) L_{q r}^{2} L_{f r} \\
& +\left(-\frac{68}{3}\right) L_{q r} L_{f r}^{2}+\left(\frac{388}{9}-32 \zeta_{3}-48 \zeta_{2}\right) L_{f r}^{2}+\left(\frac{4972}{81}\right) L_{q r}^{3}+\left(\frac{1784}{9}\right. \\
& \left.\left.+\frac{256}{9} \zeta_{2}\right) L_{q r} L_{f r}+\left(\frac{109118}{81}-\frac{4336}{27} \zeta_{3}-\frac{24656}{27} \zeta_{2}+\frac{464}{5} \zeta_{2}^{2}\right) L_{q r}+(12) L_{f r}^{3}\right) C_{F}^{2} n_{f} \\
& +\left(-\frac{61807}{729}-\frac{1136}{81} \zeta_{3}+\frac{3728}{27} \zeta_{2}+\frac{448}{135} \zeta_{2}^{2}+\left(-\frac{244}{9}+\frac{32}{9} \zeta_{2}\right) L_{q r}^{2}+\left(-\frac{8}{9}\right) L_{f r}^{3}+\left(\frac{4}{9}\right.\right. \\
& \left.+\frac{32}{9} \zeta_{2}\right) L_{f r}^{2}+\left(\frac{88}{27}\right) L_{q r}^{3}+\left(\frac{34}{9}+\frac{32}{9} \zeta_{3}-\frac{160}{27} \zeta_{2}\right) L_{f r}+\left(\frac{6556}{81}-\frac{64}{27} \zeta_{3}\right. \\
& \left.\left.-\frac{1568}{27} \zeta_{2}\right) L_{q r}\right) C_{F} n_{f}^{2}+\left(\frac{2446783}{2187}+\frac{136}{3} \zeta_{5}-\frac{37456}{81} \zeta_{3}-\frac{93268}{81} \zeta_{2}+\frac{928}{9} \zeta_{2} \zeta_{3}\right. \\
& +\frac{1612}{135} \zeta_{2}^{2}+\left(-\frac{213536}{243}+\frac{3440}{27} \zeta_{3}+\frac{12448}{27} \zeta_{2}-\frac{344}{15} \zeta_{2}^{2}\right) L_{q r}+\left(-40-\frac{400}{9} \zeta_{3}\right. \\
& \left.+\frac{2672}{27} \zeta_{2}-\frac{8}{5} \zeta_{2}^{2}\right) L_{f r}+\left(-\frac{2024}{81}\right) L_{q r}^{3}+\left(-\frac{146}{9}+16 \zeta_{3}-\frac{352}{9} \zeta_{2}\right) L_{f r}^{2}+\left(\frac{88}{9}\right) L_{f r}^{3} \\
& \left.+\left(\frac{20014}{81}-16 \zeta_{3}-\frac{352}{9} \zeta_{2}\right) L_{q r}^{2}\right) C_{A} C_{F} n_{f}+\left(\frac{65475811}{8748}+\frac{3128}{9} \zeta_{5}-\frac{546572}{81} \zeta_{3}\right. \\
& +\frac{592}{3} \zeta_{3}^{2}-\frac{503536}{81} \zeta_{2}+2208 \zeta_{2} \zeta_{3}+\frac{350464}{135} \zeta_{2}^{2}-\frac{123632}{315} \zeta_{2}^{3}+\left(-\frac{3309509}{486}+240 \zeta_{5}\right. \\
& \left.+\frac{94264}{27} \zeta_{3}+\frac{102992}{27} \zeta_{2}-352 \zeta_{2} \zeta_{3}-\frac{10208}{15} \zeta_{2}^{2}\right) L_{q r}+\left(-\frac{7844}{9}+416 \zeta_{3}\right. \\
& \left.-\frac{1408}{9} \zeta_{2}\right) L_{q r} L_{f r}+\left(-\frac{2323}{9}+32 \zeta_{3}+264 \zeta_{2}\right) L_{f r}^{2}+\left(-\frac{6358}{27}\right) L_{q r}^{3} \\
& +\left(\frac{374}{3}\right) L_{q r} L_{f r}^{2}+\left(\frac{374}{3}\right) L_{q r}^{2} L_{f r}+\left(\frac{44564}{27}-240 \zeta_{5}-\frac{6032}{3} \zeta_{3}+\frac{2708}{27} \zeta_{2}+352 \zeta_{2} \zeta_{3}\right. \\
& \left.\left.\left.-\frac{1136}{5} \zeta_{2}^{2}\right) L_{f r}+\left(\frac{57157}{27}-448 \zeta_{3}-\frac{5192}{9} \zeta_{2}\right) L_{q r}^{2}+(-66) L_{f r}^{3}\right) C_{A} C_{F}^{2}\right\} \text {. }
\end{aligned}
$$

Note that all the coefficients for the SM DY process up to third order have been obtained by some of us previously in [42].

\section{Soft-virtual coefficients at the third order}

Here we collect the new third order soft-virtual coefficients for both gluon and quark channels.

$$
\begin{aligned}
\Delta_{g g, s v}^{(3), \mathrm{GR}}= & \delta(1-z)\left\{\left(-\frac{303707}{810}-\frac{28636}{9} \zeta_{5}-\frac{186194}{135} \zeta_{3}+\frac{13216}{3} \zeta_{3}^{2}+\frac{923}{3} \zeta_{2}+\frac{8944}{3} \zeta_{2} \zeta_{3}\right.\right. \\
& +\frac{29416}{135} \zeta_{2}^{2}-\frac{64096}{105} \zeta_{2}^{3}+\left(-\frac{1319}{9}+5984 \zeta_{5}-\frac{3496}{3} \zeta_{3}+\frac{15232}{27} \zeta_{2}-3872 \zeta_{2} \zeta_{3}\right. \\
& \left.-\frac{396}{5} \zeta_{2}^{2}\right) L_{q f}+\left(\frac{110}{3}+\frac{968}{3} \zeta_{3}+\frac{736}{3} \zeta_{2}-\frac{1152}{5} \zeta_{2}^{2}\right) L_{q f}^{2}+\left(\frac{512}{3} \zeta_{3}\right. \\
& \left.\left.-\frac{352}{3} \zeta_{2}\right) L_{q f}^{3}\right) C_{A}^{3}+\left(-\frac{442583}{4374}-\frac{1136}{81} \zeta_{3}+\frac{8396}{81} \zeta_{2}-\frac{208}{15} \zeta_{2}^{2}+\left(-\frac{320}{27}\right.\right.
\end{aligned}
$$




$$
\begin{aligned}
& \left.\left.+\frac{32}{9} \zeta_{2}\right) L_{q f}^{2}+\left(\frac{88}{81}\right) L_{q f}^{3}+\left(\frac{13291}{243}+\frac{64}{9} \zeta_{3}-\frac{1024}{27} \zeta_{2}\right) L_{q f}\right) C_{A} n_{f}^{2}+\left(-\frac{46372}{729}\right. \\
& \left.-\frac{128}{81} \zeta_{3}+\frac{5504}{81} \zeta_{2}+\left(-\frac{688}{81}\right) L_{q f}^{2}+\left(\frac{64}{81}\right) L_{q f}^{3}+\left(\frac{8776}{243}-\frac{512}{27} \zeta_{2}\right) L_{q f}\right) C_{F} n_{f}^{2}+( \\
& -\frac{62429}{3645}-\frac{10592}{405} \zeta_{3}+\frac{6016}{81} \zeta_{2}+\frac{64}{15} \zeta_{2}^{2}+\left(-\frac{752}{81}\right) L_{q f}^{2}+\left(\frac{128}{81}\right) L_{q f}^{3}+\left(\frac{3932}{243}+\frac{64}{3} \zeta_{3}\right. \\
& \left.\left.-\frac{1024}{27} \zeta_{2}\right) L_{q f}\right) C_{F}^{2} n_{f}+\left(\frac{5410051}{21870}+\frac{5032}{9} \zeta_{5}+\frac{177278}{405} \zeta_{3}-\frac{20276}{81} \zeta_{2}-\frac{1840}{3} \zeta_{2} \zeta_{3}\right. \\
& +\frac{964}{27} \zeta_{2}^{2}+\left(-\frac{1046}{27}+144 \zeta_{3}+\frac{272}{3} \zeta_{2}\right) L_{q f}^{2}+\left(\frac{484}{81}-\frac{64}{3} \zeta_{2}\right) L_{q f}^{3}+\left(\frac{9029}{243}-\frac{3824}{9} \zeta_{3}\right. \\
& \left.\left.-\frac{2704}{27} \zeta_{2}+\frac{40}{3} \zeta_{2}^{2}\right) L_{q f}\right) C_{A}^{2} n_{f}+\left(\frac{1056967}{3645}-\frac{5296}{45} \zeta_{3}-\frac{4868}{27} \zeta_{2}+64 \zeta_{2} \zeta_{3}-112 \zeta_{2}^{2}\right. \\
& \left.+\left(-\frac{2722}{81}+\frac{128}{9} \zeta_{2}\right) L_{q f}^{2}+\left(-\frac{5075}{243}+\frac{80}{3} \zeta_{3}-\frac{544}{27} \zeta_{2}\right) L_{q f}+\left(\frac{176}{27}\right) L_{q f}^{3}\right) C_{A} C_{F} n_{f} \\
& +\left(\left(-\frac{464173}{3645}+80 \zeta_{5}-\frac{256}{45} \zeta_{3}+\frac{3308}{27} \zeta_{2}-\frac{320}{3} \zeta_{2} \zeta_{3}+\frac{5008}{45} \zeta_{2}^{2}+\left(-\frac{176}{27}\right) L_{q f}^{3}\right.\right. \\
& \left.+\left(\frac{2128}{81}-\frac{128}{9} \zeta_{2}\right) L_{q f}^{2}+\left(\frac{9530}{243}-\frac{80}{3} \zeta_{3}+\frac{112}{27} \zeta_{2}\right) L_{q f}\right) C_{A} C_{F} n_{f}+\left(-\frac{2410567}{21870}\right. \\
& -\frac{760}{3} \zeta_{5}-\frac{11591}{405} \zeta_{3}+\frac{4169}{81} \zeta_{2}+\frac{332}{3} \zeta_{2} \zeta_{3}-\frac{5071}{45} \zeta_{2}^{2}+\left(-\frac{484}{81}+\frac{128}{3} \zeta_{2}\right) L_{q f}^{3} \\
& \left.+\left(\frac{5092}{243}+\frac{6104}{9} \zeta_{3}+\frac{112}{9} \zeta_{2}+\frac{16}{15} \zeta_{2}^{2}\right) L_{q f}+\left(\frac{668}{27}-\frac{608}{3} \zeta_{3}-144 \zeta_{2}\right) L_{q f}^{2}\right) C_{A}^{2} n_{f}+( \\
& -\frac{35176}{3645}+160 \zeta_{5}-\frac{29368}{405} \zeta_{3}-\frac{6016}{81} \zeta_{2}-\frac{64}{15} \zeta_{2}^{2}+\left(-\frac{3446}{243}-\frac{64}{3} \zeta_{3}+\frac{1024}{27} \zeta_{2}\right) L_{q f} \\
& \left.+\left(-\frac{128}{81}\right) L_{q f}^{3}+\left(\frac{752}{81}\right) L_{q f}^{2}\right) C_{F}^{2} n_{f}+\left(\frac{16087}{486}+\frac{3296}{81} \zeta_{3}-\frac{4216}{81} \zeta_{2}+\left(-\frac{11186}{243}\right.\right. \\
& \left.\left.-\frac{32}{3} \zeta_{3}+\frac{128}{9} \zeta_{2}\right) L_{q f}+\left(-\frac{160}{81}\right) L_{q f}^{3}+\left(\frac{1340}{81}\right) L_{q f}^{2}\right) C_{F} n_{f}^{2}+\left(\frac{1404698}{10935}-\frac{10952}{405} \zeta_{3}\right. \\
& -\frac{2104}{27} \zeta_{2}-\frac{32}{45} \zeta_{2}^{2}+\left(-\frac{23468}{243}+\frac{32}{9} \zeta_{3}+\frac{1348}{27} \zeta_{2}\right) L_{q f}+\left(-\frac{220}{81}\right) L_{q f}^{3}+\left(\frac{2125}{81}\right. \\
& \left.\left.\left.-\frac{64}{9} \zeta_{2}\right) L_{q f}^{2}\right) C_{A} n_{f}^{2}\right) r_{g}+\left(\left(-\frac{55825}{1458}+\frac{280}{9} \zeta_{3}+\frac{973}{81} \zeta_{2}+\frac{64}{3} \zeta_{2}^{2}+\left(-\frac{1057}{81}\right.\right.\right. \\
& \left.\left.+\frac{32}{9} \zeta_{2}\right) L_{q f}^{2}+\left(\frac{44}{27}\right) L_{q f}^{3}+\left(\frac{9070}{243}-\frac{32}{3} \zeta_{3}-12 \zeta_{2}\right) L_{q f}\right) C_{A} n_{f}^{2}+\left(\frac{10465}{729}-\frac{280}{9} \zeta_{3}\right. \\
& \left.\left.\left.+\frac{656}{81} \zeta_{2}+\left(-\frac{544}{81}\right) L_{q f}^{2}+\left(\frac{32}{27}\right) L_{q f}^{3}+\left(\frac{1114}{243}+\frac{32}{3} \zeta_{3}+\frac{128}{27} \zeta_{2}\right) L_{q f}\right) C_{F} n_{f}^{2}\right) r_{g}^{2}\right\} \\
& +\mathcal{D}_{5}\left\{(512) C_{A}^{3}\right\}+\mathcal{D}_{4}\left\{\left(-\frac{7040}{9}+(1280) L_{q f}\right) C_{A}^{3}+\left(\frac{1280}{9}\right) C_{A}^{2} n_{f}\right\}+\mathcal{D}_{3}\{( \\
& \left.-\frac{18752}{27}-3584 \zeta_{2}+\left(-\frac{5632}{9}\right) L_{q f}+(1024) L_{q f}^{2}\right) C_{A}^{3}+\left(-\frac{10496}{27}\right. \\
& \left.\left.+\left(\frac{2560}{9}\right) L_{q f}\right) C_{A}^{2} n_{f}+\left(\frac{256}{27}\right) C_{A} n_{f}^{2}+\left(\left(\frac{4480}{9}+\left(-\frac{512}{3}\right) L_{q f}\right) C_{A}^{2} n_{f}\right) r_{g}\right\}
\end{aligned}
$$




$$
\begin{aligned}
& +\mathcal{D}_{2}\left\{\left(-1168+11584 \zeta_{3}+\frac{11968}{3} \zeta_{2}+\left(-1472-5376 \zeta_{2}\right) L_{q f}+(256) L_{q f}^{3}\right.\right. \\
& \left.+(352) L_{q f}^{2}\right) C_{A}^{3}+\left(-\frac{640}{27}+\left(\frac{128}{9}\right) L_{q f}\right) C_{A} n_{f}^{2}+\left(\frac{8128}{27}-\frac{2176}{3} \zeta_{2}+(\right. \\
& \left.\left.-\frac{4544}{9}\right) L_{q f}+(192) L_{q f}^{2}\right) C_{A}^{2} n_{f}+\left(\left(-\frac{6160}{27}+\left(\frac{7424}{9}\right) L_{q f}\right.\right. \\
& \left.\left.\left.+(-256) L_{q f}^{2}\right) C_{A}^{2} n_{f}+\left(\frac{1120}{27}+\left(-\frac{128}{9}\right) L_{q f}\right) C_{A} n_{f}^{2}\right) r_{g}+(32) C_{A} C_{F} n_{f}\right\} \\
& +\mathcal{D}_{1}\left\{\left(-\frac{6932}{9}-\frac{20416}{3} \zeta_{3}+\frac{17120}{9} \zeta_{2}-\frac{9856}{5} \zeta_{2}^{2}+\left(-\frac{21536}{27}+11520 \zeta_{3}\right.\right.\right. \\
& \left.\left.+\frac{5632}{3} \zeta_{2}\right) L_{q f}+\left(-\frac{1472}{3}-2304 \zeta_{2}\right) L_{q f}^{2}+\left(\frac{704}{3}\right) L_{q f}^{3}\right) C_{A}^{3}+\left(\frac{1600}{81}-\frac{256}{9} \zeta_{2}+(\right. \\
& \left.\left.-\frac{640}{27}\right) L_{q f}+\left(\frac{64}{9}\right) L_{q f}^{2}\right) C_{A} n_{f}^{2}+\left(\frac{4904}{81}+128 \zeta_{3}-\frac{2048}{9} \zeta_{2}+\left(-\frac{992}{9}\right) L_{q f}\right. \\
& \left.+\left(\frac{256}{9}\right) L_{q f}^{2}\right) C_{A} C_{F} n_{f}+\left(\frac{58160}{81}+\frac{3328}{3} \zeta_{3}+\frac{3200}{9} \zeta_{2}+\left(-\frac{4864}{27}-\frac{1664}{3} \zeta_{2}\right) L_{q f}\right. \\
& \left.+\left(-\frac{928}{9}\right) L_{q f}^{2}+\left(\frac{128}{3}\right) L_{q f}^{3}\right) C_{A}^{2} n_{f}+\left(\left(-\frac{44840}{81}+256 \zeta_{3}-832 \zeta_{2}+\left(-\frac{256}{3}\right) L_{q f}^{3}\right.\right. \\
& \left.+\left(\frac{1888}{9}\right) L_{q f}^{2}+\left(\frac{2528}{9}+\frac{640}{3} \zeta_{2}\right) L_{q f}\right) C_{A}^{2} n_{f}+\left(-\frac{5600}{81}+\left(-\frac{128}{9}\right) L_{q f}^{2}\right. \\
& \left.+\left(\frac{1760}{27}\right) L_{q f}\right) C_{A} n_{f}^{2}+\left(\frac{9568}{81}-256 \zeta_{3}+\frac{2048}{9} \zeta_{2}+\left(-\frac{256}{9}\right) L_{q f}^{2}\right. \\
& \left.\left.+\left(\frac{704}{9}\right) L_{q f}\right) C_{A} C_{F} n_{f}\right) r_{g}+\left(\left(\frac{4900}{81}+\frac{128}{3} \zeta_{2}+\left(-\frac{1120}{27}\right) L_{q f}\right.\right. \\
& \left.\left.\left.+\left(\frac{64}{9}\right) L_{q f}^{2}\right) C_{A} n_{f}^{2}\right) r_{g}^{2}\right\}+\mathcal{D}_{0}\left\{\left(-\frac{11164}{729}-\frac{7600}{9} \zeta_{3}-\frac{15280}{81} \zeta_{2}-\frac{544}{15} \zeta_{2}^{2}+(\right.\right. \\
& \left.\left.-\frac{4912}{27}-\frac{224}{3} \zeta_{2}\right) L_{q f}^{2}+\left(\frac{704}{27}\right) L_{q f}^{3}+\left(\frac{11336}{27}+\frac{1664}{3} \zeta_{3}+\frac{896}{9} \zeta_{2}\right) L_{q f}\right) C_{A}^{2} n_{f}+( \\
& \left.-\frac{3712}{729}+\frac{320}{27} \zeta_{3}+\frac{640}{27} \zeta_{2}+\left(-\frac{160}{27}\right) L_{q f}^{2}+\left(\frac{32}{27}\right) L_{q f}^{3}+\left(\frac{800}{81}-\frac{128}{9} \zeta_{2}\right) L_{q f}\right) C_{A} n_{f}^{2} \\
& +\left(\frac{3422}{27}-\frac{608}{9} \zeta_{3}-32 \zeta_{2}-\frac{64}{5} \zeta_{2}^{2}+\left(-\frac{568}{9}\right) L_{q f}^{2}+\left(\frac{128}{9}\right) L_{q f}^{3}+\left(\frac{2452}{81}+64 \zeta_{3}\right.\right. \\
& \left.\left.-\frac{1024}{9} \zeta_{2}\right) L_{q f}\right) C_{A} C_{F} n_{f}+\left(\frac{390086}{729}+11904 \zeta_{5}-\frac{46952}{27} \zeta_{3}+\frac{29824}{81} \zeta_{2}-\frac{23200}{3} \zeta_{2} \zeta_{3}\right. \\
& +\frac{4048}{15} \zeta_{2}^{2}+\left(-\frac{66746}{81}-\frac{3344}{3} \zeta_{3}+\frac{4144}{3} \zeta_{2}-\frac{4928}{5} \zeta_{2}^{2}\right) L_{q f}+\left(\frac{116}{9}+2240 \zeta_{3}\right. \\
& \left.\left.-176 \zeta_{2}\right) L_{q f}^{2}+\left(\frac{968}{27}-256 \zeta_{2}\right) L_{q f}^{3}\right) C_{A}^{3}+\left(\left(-\frac{56560}{243}+\frac{3640}{3} \zeta_{3}+\frac{6160}{27} \zeta_{2}+(\right.\right. \\
& \left.\left.-\frac{15956}{81}-288 \zeta_{3}-\frac{4448}{9} \zeta_{2}\right) L_{q f}+\left(-\frac{352}{9}\right) L_{q f}^{3}+\left(\frac{5332}{27}+\frac{320}{3} \zeta_{2}\right) L_{q f}^{2}\right) C_{A}^{2} n_{f} \\
& +\left(\frac{7840}{243}-\frac{1120}{27} \zeta_{2}+\left(-\frac{1232}{27}+\frac{128}{9} \zeta_{2}\right) L_{q f}+\left(-\frac{32}{9}\right) L_{q f}^{3}+\left(\frac{200}{9}\right) L_{q f}^{2}\right) C_{A} n_{f}^{2}
\end{aligned}
$$




$$
\begin{aligned}
& \left.+\left(\left(-\frac{128}{9}\right) L_{q f}^{3}+\left(\frac{352}{9}\right) L_{q f}^{2}+\left(\frac{4784}{81}-128 \zeta_{3}+\frac{1024}{9} \zeta_{2}\right) L_{q f}\right) C_{A} C_{F} n_{f}\right) r_{g} \\
& \left.+\left(\left(\left(-\frac{560}{27}\right) L_{q f}^{2}+\left(\frac{32}{9}\right) L_{q f}^{3}+\left(\frac{2450}{81}+\frac{64}{3} \zeta_{2}\right) L_{q f}\right) C_{A} n_{f}^{2}\right) r_{g}^{2}\right\} .
\end{aligned}
$$

$$
\begin{aligned}
& \Delta_{q \bar{q}, s v}^{(3), \mathrm{GR}}=\delta(1-z)\left\{\left(-\frac{6131417}{1458}+\frac{3344}{3} \zeta_{5}+\frac{188276}{81} \zeta_{3}+\frac{10336}{3} \zeta_{3}^{2}+\frac{8122}{9} \zeta_{2}-432 \zeta_{2} \zeta_{3}\right.\right. \\
& +\frac{4156}{45} \zeta_{2}^{2}-\frac{184736}{315} \zeta_{2}^{3}+\left(-\frac{49402}{27}+\frac{5984}{3} \zeta_{3}+\frac{10112}{9} \zeta_{2}-\frac{1792}{5} \zeta_{2}^{2}\right) L_{q f}^{2}+\left(\frac{19652}{81}\right. \\
& \left.+\frac{512}{3} \zeta_{3}-\frac{1088}{3} \zeta_{2}\right) L_{q f}^{3}+\left(\frac{760175}{162}+5664 \zeta_{5}-\frac{54304}{9} \zeta_{3}-\frac{26584}{27} \zeta_{2}-2752 \zeta_{2} \zeta_{3}\right. \\
& \left.\left.-\frac{176}{3} \zeta_{2}^{2}\right) L_{q f}\right) C_{F}^{3}+\left(-\frac{31255393}{8748}-\frac{2852}{3} \zeta_{5}+\frac{84131}{27} \zeta_{3}-\frac{400}{3} \zeta_{3}^{2}+\frac{39781}{27} \zeta_{2}\right. \\
& -\frac{820}{3} \zeta_{2} \zeta_{3}+\frac{20323}{135} \zeta_{2}^{2}+\frac{13264}{315} \zeta_{2}^{3}+\left(-\frac{15055}{27}+88 \zeta_{3}\right) L_{q f}^{2}+\left(\frac{4114}{81}\right) L_{q f}^{3}+\left(\frac{561610}{243}\right. \\
& \left.\left.+80 \zeta_{5}-\frac{8792}{9} \zeta_{3}-\frac{11056}{27} \zeta_{2}+\frac{68}{5} \zeta_{2}^{2}\right) L_{q f}\right) C_{A}^{2} C_{F}+\left(-\frac{2467183}{2187}+\frac{5536}{9} \zeta_{5}+\frac{38488}{81} \zeta_{3}\right. \\
& +\frac{66184}{81} \zeta_{2}-\frac{5504}{9} \zeta_{2} \zeta_{3}-\frac{21808}{135} \zeta_{2}^{2}+\left(-\frac{40028}{81}+160 \zeta_{3}+\frac{896}{9} \zeta_{2}\right) L_{q f}^{2}+\left(\frac{4972}{81}\right. \\
& \left.\left.-\frac{64}{3} \zeta_{2}\right) L_{q f}^{3}+\left(\frac{109118}{81}-\frac{3280}{9} \zeta_{3}-\frac{13328}{27} \zeta_{2}+\frac{112}{15} \zeta_{2}^{2}\right) L_{q f}\right) C_{F}^{2} n_{f}+\left(-\frac{61807}{729}\right. \\
& -\frac{2416}{81} \zeta_{3}+\frac{10384}{81} \zeta_{2}+\frac{128}{27} \zeta_{2}^{2}+\left(-\frac{244}{9}\right) L_{q f}^{2}+\left(\frac{88}{27}\right) L_{q f}^{3}+\left(\frac{6556}{81}+\frac{64}{9} \zeta_{3}\right. \\
& \left.\left.-\frac{416}{9} \zeta_{2}\right) L_{q f}\right) C_{F} n_{f}^{2}+\left(\frac{2446783}{2187}+\frac{136}{3} \zeta_{5}-\frac{6320}{27} \zeta_{3}-\frac{8540}{9} \zeta_{2}+\frac{224}{3} \zeta_{2} \zeta_{3}-\frac{1060}{27} \zeta_{2}^{2}\right. \\
& +\left(-\frac{213536}{243}+\frac{208}{9} \zeta_{3}+\frac{2608}{9} \zeta_{2}-\frac{8}{5} \zeta_{2}^{2}\right) L_{q f}+\left(-\frac{2024}{81}\right) L_{q f}^{3}+\left(\frac{20014}{81}\right. \\
& \left.\left.-16 \zeta_{3}\right) L_{q f}^{2}\right) C_{A} C_{F} n_{f}+\left(\frac{65475811}{8748}-\frac{30664}{9} \zeta_{5}-\frac{178948}{27} \zeta_{3}+\frac{3280}{3} \zeta_{3}^{2}-\frac{201062}{81} \zeta_{2}\right. \\
& +\frac{32960}{9} \zeta_{2} \zeta_{3}+\frac{40576}{135} \zeta_{2}^{2}-\frac{20816}{315} \zeta_{2}^{3}+\left(-\frac{3309509}{486}+240 \zeta_{5}+\frac{44584}{9} \zeta_{3}+\frac{46808}{27} \zeta_{2}\right. \\
& \left.-1120 \zeta_{2} \zeta_{3}-\frac{448}{15} \zeta_{2}^{2}\right) L_{q f}+\left(-\frac{6358}{27}+\frac{352}{3} \zeta_{2}\right) L_{q f}^{3}+\left(\frac{57157}{27}-1152 \zeta_{3}-\frac{1664}{3} \zeta_{2}\right. \\
& \left.\left.+128 \zeta_{2}^{2}\right) L_{q f}^{2}\right) C_{A} C_{F}^{2}+\left(\left(-\frac{10514857}{2187}+\frac{1280}{3} \zeta_{5}+\frac{6784}{27} \zeta_{3}+\frac{19088}{9} \zeta_{2}+\frac{256}{3} \zeta_{2} \zeta_{3}\right.\right. \\
& -\frac{22288}{45} \zeta_{2}^{2}+\left(-\frac{131140}{81}+128 \zeta_{3}+\frac{704}{9} \zeta_{2}\right) L_{q f}^{2}+\left(\frac{1760}{9}\right) L_{q f}^{3}+\left(\frac{1178048}{243}-544 \zeta_{3}\right. \\
& \left.\left.-\frac{10480}{9} \zeta_{2}+\frac{64}{5} \zeta_{2}^{2}\right) L_{q f}\right) C_{A} C_{F}^{2}+\left(-\frac{1268944}{2187}-\frac{10528}{81} \zeta_{3}+\frac{60512}{81} \zeta_{2}-\frac{896}{45} \zeta_{2}^{2}+(\right. \\
& \left.\left.-\frac{10780}{81}\right) L_{q f}^{2}+\left(\frac{1232}{81}\right) L_{q f}^{3}+\left(\frac{107948}{243}+\frac{256}{3} \zeta_{3}-\frac{784}{3} \zeta_{2}\right) L_{q f}\right) C_{A} C_{F} n_{f}+\left(\frac{39772}{729}\right. \\
& \left.-\frac{320}{3} \zeta_{2}+\left(-\frac{4096}{81}+\frac{128}{3} \zeta_{2}\right) L_{q f}+\left(-\frac{64}{27}\right) L_{q f}^{3}+\left(\frac{160}{9}\right) L_{q f}^{2}\right) C_{F} n_{f}^{2}+\left(\frac{2196934}{2187}\right.
\end{aligned}
$$




$$
\begin{aligned}
& +\frac{17312}{81} \zeta_{3}-\frac{53440}{81} \zeta_{2}+\frac{2816}{15} \zeta_{2}^{2}+\left(-\frac{280736}{243}-128 \zeta_{3}+\frac{9856}{27} \zeta_{2}\right) L_{q f}+( \\
& \left.\left.-\frac{4768}{81}\right) L_{q f}^{3}+\left(\frac{35560}{81}-\frac{256}{9} \zeta_{2}\right) L_{q f}^{2}\right) C_{F}^{2} n_{f}+\left(\frac{3381256}{2187}-480 \zeta_{5}+\frac{29972}{81} \zeta_{3}\right. \\
& -\frac{3344}{3} \zeta_{2}-\frac{208}{3} \zeta_{2} \zeta_{3}+\frac{2324}{45} \zeta_{2}^{2}+\left(-\frac{234820}{243}-\frac{128}{3} \zeta_{3}+\frac{10384}{27} \zeta_{2}\right) L_{q f}+( \\
& \left.\left.-\frac{1936}{81}\right) L_{q f}^{3}+\left(\frac{6316}{27}\right) L_{q f}^{2}\right) C_{A}^{2} C_{F}+\left(\frac{263089}{81}-\frac{72176}{81} \zeta_{3}-\frac{22984}{81} \zeta_{2}-\frac{11488}{45} \zeta_{2}^{2}+(\right. \\
& \left.-\frac{1041604}{243}+\frac{17152}{9} \zeta_{3}+\frac{25216}{27} \zeta_{2}-\frac{128}{15} \zeta_{2}^{2}\right) L_{q f}+\left(-\frac{21728}{81}+\frac{512}{3} \zeta_{2}\right) L_{q f}^{3} \\
& \left.\left.+\left(\frac{150136}{81}-\frac{2816}{3} \zeta_{3}-\frac{4864}{9} \zeta_{2}\right) L_{q f}^{2}\right) C_{F}^{3}\right) r_{q}+\left(\left(-\frac{325244}{729}-\frac{79328}{81} \zeta_{2}+\frac{1024}{3} \zeta_{2}^{2}\right.\right. \\
& \left.+\left(-\frac{29632}{81}+\frac{512}{9} \zeta_{2}\right) L_{q f}^{2}+\left(\frac{1664}{27}\right) L_{q f}^{3}+\left(\frac{172408}{243}+\frac{512}{3} \zeta_{2}\right) L_{q f}\right) C_{F}^{3}+\left(-\frac{79288}{729}\right. \\
& \left.-\frac{5504}{27} \zeta_{2}+\left(-\frac{4928}{81}\right) L_{q f}^{2}+\left(\frac{256}{27}\right) L_{q f}^{3}+\left(\frac{34976}{243}+\frac{512}{9} \zeta_{2}\right) L_{q f}\right) C_{F}^{2} n_{f}+\left(\frac{266084}{729}\right. \\
& +\frac{55952}{81} \zeta_{2}+\left(-\frac{113608}{243}-\frac{4672}{27} \zeta_{2}\right) L_{q f}+\left(-\frac{704}{27}\right) L_{q f}^{3} \\
& \left.\left.\left.+\left(\frac{14992}{81}\right) L_{q f}^{2}\right) C_{A} C_{F}^{2}\right) r_{q}^{2}\right\}+\mathcal{D}_{5}\left\{(512) C_{F}^{3}\right\}+\mathcal{D}_{4}\left\{\left(-\frac{7040}{9}\right) C_{A} C_{F}^{2}\right. \\
& \left.+\left(\frac{1280}{9}\right) C_{F}^{2} n_{f}+\left((1280) L_{q f}\right) C_{F}^{3}\right\}+\mathcal{D}_{3}\left\{\left(-\frac{31744}{9}-3072 \zeta_{2}+\left(\frac{4352}{3}\right) L_{q f}\right.\right. \\
& \left.+(1024) L_{q f}^{2}\right) C_{F}^{3}+\left(-\frac{2560}{9}+\left(\frac{2560}{9}\right) L_{q f}\right) C_{F}^{2} n_{f}+\left(-\frac{2816}{27}\right) C_{A} C_{F} n_{f} \\
& +\left(\frac{256}{27}\right) C_{F} n_{f}^{2}+\left(\frac{7744}{27}\right) C_{A}^{2} C_{F}+\left(\frac{17152}{9}-512 \zeta_{2}+\left(-\frac{14080}{9}\right) L_{q f}\right) C_{A} C_{F}^{2} \\
& \left.+\left(\left(\frac{8704}{9}+\left(-\frac{2048}{3}\right) L_{q f}\right) C_{F}^{3}\right) r_{q}\right\}+\mathcal{D}_{2}\left\{\left(-\frac{28480}{27}+\frac{704}{3} \zeta_{2}\right.\right. \\
& \left.+\left(\frac{3872}{9}\right) L_{q f}\right) C_{A}^{2} C_{F}+\left(-\frac{1696}{27}-\frac{2048}{3} \zeta_{2}+\left(-\frac{2752}{9}\right) L_{q f}+(192) L_{q f}^{2}\right) C_{F}^{2} n_{f} \\
& +\left(-\frac{640}{27}+\left(\frac{128}{9}\right) L_{q f}\right) C_{F} n_{f}^{2}+\left(\frac{4864}{27}+1344 \zeta_{3}+\frac{11264}{3} \zeta_{2}+\left(\frac{19744}{9}-768 \zeta_{2}\right) L_{q f}\right. \\
& \left.+(-1056) L_{q f}^{2}\right) C_{A} C_{F}^{2}+\left(\frac{9248}{27}-\frac{128}{3} \zeta_{2}+\left(-\frac{1408}{9}\right) L_{q f}\right) C_{A} C_{F} n_{f}+\left(10240 \zeta_{3}\right. \\
& \left.+\left(-\frac{15872}{3}-4608 \zeta_{2}\right) L_{q f}+(256) L_{q f}^{3}+(2176) L_{q f}^{2}\right) C_{F}^{3}+\left(\left(-\frac{11968}{27}\right.\right. \\
& \left.+\left(\frac{2816}{9}\right) L_{q f}\right) C_{A} C_{F}^{2}+\left(\frac{2176}{27}+\left(-\frac{512}{9}\right) L_{q f}\right) C_{F}^{2} n_{f}+\left(\left(\frac{4352}{3}\right) L_{q f}\right. \\
& \left.\left.\left.+(-1024) L_{q f}^{2}\right) C_{F}^{3}\right) r_{q}\right\}+\mathcal{D}_{1}\left\{\left(-\frac{604948}{81}-4160 \zeta_{3}-\frac{1664}{3} \zeta_{2}+\frac{3648}{5} \zeta_{2}^{2}+(\right.\right. \\
& \left.\left.-\frac{704}{3}\right) L_{q f}^{3}+\left(-\frac{400}{9}-256 \zeta_{2}\right) L_{q f}^{2}+\left(\frac{37456}{9}+512 \zeta_{3}+\frac{8768}{3} \zeta_{2}\right) L_{q f}\right) C_{A} C_{F}^{2}+(
\end{aligned}
$$




$$
\begin{aligned}
& \left.-\frac{32816}{81}+384 \zeta_{2}+\left(-\frac{704}{9}\right) L_{q f}^{2}+\left(\frac{9248}{27}-\frac{128}{3} \zeta_{2}\right) L_{q f}\right) C_{A} C_{F} n_{f}+\left(\frac{1600}{81}-\frac{256}{9} \zeta_{2}\right. \\
& \left.+\left(-\frac{640}{27}\right) L_{q f}+\left(\frac{64}{9}\right) L_{q f}^{2}\right) C_{F} n_{f}^{2}+\left(\frac{32768}{27}+1280 \zeta_{3}-\frac{256}{9} \zeta_{2}+\left(-\frac{7552}{9}\right.\right. \\
& \left.\left.-\frac{1792}{3} \zeta_{2}\right) L_{q f}+\left(\frac{128}{3}\right) L_{q f}^{3}+\left(\frac{608}{9}\right) L_{q f}^{2}\right) C_{F}^{2} n_{f}+\left(\frac{124024}{81}-704 \zeta_{3}-\frac{12032}{9} \zeta_{2}\right. \\
& \left.+\frac{704}{5} \zeta_{2}^{2}+\left(-\frac{28480}{27}+\frac{704}{3} \zeta_{2}\right) L_{q f}+\left(\frac{1936}{9}\right) L_{q f}^{2}\right) C_{A}^{2} C_{F}+\left(\frac{172372}{27}-1984 \zeta_{3}\right. \\
& +\frac{42656}{9} \zeta_{2}-\frac{14208}{5} \zeta_{2}^{2}+\left(-\frac{137200}{27}+11008 \zeta_{3}-\frac{5504}{3} \zeta_{2}\right) L_{q f}+(-736 \\
& \left.\left.-2048 \zeta_{2}\right) L_{q f}^{2}+\left(\frac{2176}{3}\right) L_{q f}^{3}\right) C_{F}^{3}+\left(\left(-\frac{287968}{81}-\frac{12800}{9} \zeta_{2}+\left(-\frac{3328}{9}\right) L_{q f}^{2}+(\right.\right. \\
& \left.\left.-\frac{1024}{3}\right) L_{q f}^{3}+\left(\frac{95360}{27}+\frac{2048}{3} \zeta_{2}\right) L_{q f}\right) C_{F}^{3}+\left(-\frac{16064}{27}+\frac{1024}{3} \zeta_{2}+\left(-\frac{1024}{9}\right) L_{q f}^{2}\right. \\
& \left.+\left(\frac{12416}{27}\right) L_{q f}\right) C_{F}^{2} n_{f}+\left(\frac{198112}{81}-\frac{9728}{9} \zeta_{2}+\left(-\frac{53120}{27}+\frac{512}{3} \zeta_{2}\right) L_{q f}\right. \\
& \left.\left.+\left(\frac{1408}{3}\right) L_{q f}^{2}\right) C_{A} C_{F}^{2}\right) r_{q}+\left(\left(\frac{18496}{81}+\frac{2048}{3} \zeta_{2}+\left(-\frac{8704}{27}\right) L_{q f}\right.\right. \\
& \left.\left.\left.+\left(\frac{1024}{9}\right) L_{q f}^{2}\right) C_{F}^{3}\right) r_{q}^{2}\right\}+\mathcal{D}_{0}\left\{\left(-\frac{594058}{729}-384 \zeta_{5}+\frac{40144}{27} \zeta_{3}+\frac{98224}{81} \zeta_{2}-\frac{352}{3} \zeta_{2} \zeta_{3}\right.\right. \\
& -\frac{2992}{15} \zeta_{2}^{2}+\left(-\frac{7120}{27}+\frac{176}{3} \zeta_{2}\right) L_{q f}^{2}+\left(\frac{968}{27}\right) L_{q f}^{3}+\left(\frac{62012}{81}-352 \zeta_{3}-\frac{6016}{9} \zeta_{2}\right. \\
& \left.\left.+\frac{352}{5} \zeta_{2}^{2}\right) L_{q f}\right) C_{A}^{2} C_{F}+\left(-\frac{24754}{243}-\frac{5728}{9} \zeta_{3}+\frac{1760}{9} \zeta_{2}-\frac{1472}{15} \zeta_{2}^{2}+\left(-\frac{3784}{9}\right.\right. \\
& \left.\left.-\frac{320}{3} \zeta_{2}\right) L_{q f}^{2}+\left(\frac{224}{3}\right) L_{q f}^{3}+\left(\frac{56768}{81}+640 \zeta_{3}-\frac{1216}{9} \zeta_{2}\right) L_{q f}\right) C_{F}^{2} n_{f}+\left(-\frac{3712}{729}\right. \\
& \left.+\frac{320}{27} \zeta_{3}+\frac{640}{27} \zeta_{2}+\left(-\frac{160}{27}\right) L_{q f}^{2}+\left(\frac{32}{27}\right) L_{q f}^{3}+\left(\frac{800}{81}-\frac{128}{9} \zeta_{2}\right) L_{q f}\right) C_{F} n_{f}^{2} \\
& +\left(\frac{125252}{729}-\frac{2480}{9} \zeta_{3}-\frac{29392}{81} \zeta_{2}+\frac{736}{15} \zeta_{2}^{2}+\left(-\frac{16408}{81}+192 \zeta_{2}\right) L_{q f}+\left(-\frac{352}{27}\right) L_{q f}^{3}\right. \\
& \left.+\left(\frac{2312}{27}-\frac{32}{3} \zeta_{2}\right) L_{q f}^{2}\right) C_{A} C_{F} n_{f}+\left(\frac{400768}{243}+\frac{20416}{9} \zeta_{3}-\frac{10240}{9} \zeta_{2}-1472 \zeta_{2} \zeta_{3}\right. \\
& +\frac{1408}{3} \zeta_{2}^{2}+\left(-\frac{357418}{81}-\frac{4336}{3} \zeta_{3}+\frac{3488}{9} \zeta_{2}+\frac{1824}{5} \zeta_{2}^{2}\right) L_{q f}+\left(-\frac{2992}{9}\right) L_{q f}^{3} \\
& \left.+\left(\frac{19400}{9}-192 \zeta_{3}+\frac{1216}{3} \zeta_{2}\right) L_{q f}^{2}\right) C_{A} C_{F}^{2}+\left(12288 \zeta_{5}-\frac{63488}{9} \zeta_{3}-6144 \zeta_{2} \zeta_{3}+(\right. \\
& \left.-\frac{68600}{27}+2432 \zeta_{3}-\frac{2752}{3} \zeta_{2}\right) L_{q f}^{2}+\left(\frac{4624}{9}-256 \zeta_{2}\right) L_{q f}^{3}+\left(\frac{86186}{27}+\frac{5728}{3} \zeta_{3}\right. \\
& \left.\left.+\frac{21328}{9} \zeta_{2}-\frac{7104}{5} \zeta_{2}^{2}\right) L_{q f}\right) C_{F}^{3}+\left(\left(-\frac{109888}{243}+\frac{3808}{9} \zeta_{3}+\frac{11968}{27} \zeta_{2}+\left(-\frac{7856}{9}\right.\right.\right. \\
& \left.\left.+\frac{256}{3} \zeta_{2}\right) L_{q f}^{2}+\left(\frac{1408}{9}\right) L_{q f}^{3}+\left(\frac{124912}{81}-\frac{896}{3} \zeta_{3}-\frac{2560}{3} \zeta_{2}\right) L_{q f}\right) C_{A} C_{F}^{2}+\left(\frac{15232}{243}\right.
\end{aligned}
$$




$$
\begin{aligned}
& \left.-\frac{2176}{27} \zeta_{2}+\left(-\frac{27680}{81}+\frac{2048}{9} \zeta_{2}\right) L_{q f}+\left(-\frac{128}{3}\right) L_{q f}^{3}+\left(\frac{1888}{9}\right) L_{q f}^{2}\right) C_{F}^{2} n_{f} \\
& +\left(\frac{17408}{9} \zeta_{3}+\left(-\frac{143984}{81}-\frac{4096}{3} \zeta_{3}-\frac{6400}{9} \zeta_{2}\right) L_{q f}+\left(-\frac{1280}{3}\right) L_{q f}^{3}+\left(\frac{47680}{27}\right.\right. \\
& \left.\left.\left.+\frac{1024}{3} \zeta_{2}\right) L_{q f}^{2}\right) C_{F}^{3}\right) r_{q}+\left(\left(\left(-\frac{4352}{27}\right) L_{q f}^{2}+\left(\frac{512}{9}\right) L_{q f}^{3}+\left(\frac{9248}{81}\right.\right.\right. \\
& \left.\left.\left.\left.+\frac{1024}{3} \zeta_{2}\right) L_{q f}\right) C_{F}^{3}\right) r_{q}^{2}\right\}
\end{aligned}
$$

Open Access. This article is distributed under the terms of the Creative Commons Attribution License (CC-BY 4.0), which permits any use, distribution and reproduction in any medium, provided the original author(s) and source are credited.

\section{References}

[1] ATLAS collaboration, Observation of a new particle in the search for the Standard Model Higgs boson with the ATLAS detector at the LHC, Phys. Lett. B 716 (2012) 1 [arXiv: 1207.7214] [INSPIRE].

[2] CMS collaboration, Observation of a New Boson at a Mass of $125 \mathrm{GeV}$ with the CMS Experiment at the LHC, Phys. Lett. B 716 (2012) 30 [arXiv:1207.7235] [InSPIRE].

[3] Particle Data Group collaboration, Review of Particle Physics, PTEP 2020 (2020) $083 \mathrm{C} 01$ [INSPIRE].

[4] ATLAS collaboration, Search for new high-mass phenomena in the dilepton final state using $36 \mathrm{fb}^{-1}$ of proton-proton collision data at $\sqrt{\mathrm{s}}=13 \mathrm{TeV}$ with the ATLAS detector, JHEP 10 (2017) 182 [arXiv:1707.02424] [INSPIRE].

[5] CMS collaboration, Search for contact interactions and large extra dimensions in the dilepton mass spectra from proton-proton collisions at $\sqrt{s}=13 \mathrm{TeV}$, JHEP 04 (2019) 114 [arXiv: 1812.10443] [INSPIRE].

[6] CMS collaboration, Search for narrow resonances in dilepton mass spectra in proton-proton collisions at $\sqrt{s}=13 \mathrm{TeV}$ and combination with $8 \mathrm{TeV}$ data, Phys. Lett. B 768 (2017) 57 [arXiv: 1609.05391] [INSPIRE].

[7] C. Anastasiou, C. Duhr, F. Dulat, F. Herzog and B. Mistlberger, Higgs Boson Gluon-Fusion Production in QCD at Three Loops, Phys. Rev. Lett. 114 (2015) 212001 [arXiv:1503.06056] [INSPIRE].

[8] C. Duhr, F. Dulat and B. Mistlberger, Drell-Yan Cross Section to Third Order in the Strong Coupling Constant, Phys. Rev. Lett. 125 (2020) 172001 [arXiv:2001.07717] [InSPIRE].

[9] C. Duhr, F. Dulat and B. Mistlberger, Charged current Drell-Yan production at $N^{3} L O$, JHEP 11 (2020) 143 [arXiv:2007.13313] [INSPIRE].

[10] L. Randall and R. Sundrum, A large mass hierarchy from a small extra dimension, Phys. Rev. Lett. 83 (1999) 3370 [hep-ph/9905221] [INSPIRE].

[11] P. Artoisenet et al., A framework for Higgs characterisation, JHEP 11 (2013) 043 [arXiv: 1306.6464] [INSPIRE]. 
[12] G. Das, C. Degrande, V. Hirschi, F. Maltoni and H.-S. Shao, NLO predictions for the production of a spin-two particle at the LHC, Phys. Lett. B 770 (2017) 507 [arXiv: 1605.09359] [INSPIRE].

[13] A. Alloul, N.D. Christensen, C. Degrande, C. Duhr and B. Fuks, FeynRules $2.0-A$ complete toolbox for tree-level phenomenology, Comput. Phys. Commun. 185 (2014) 2250 [arXiv:1310.1921] [INSPIRE].

[14] J. Alwall et al., The automated computation of tree-level and next-to-leading order differential cross sections, and their matching to parton shower simulations, JHEP 07 (2014) 079 [arXiv: 1405.0301] [INSPIRE].

[15] P. Banerjee, P.K. Dhani, M.C. Kumar, P. Mathews and V. Ravindran, NNLO QCD corrections to production of a spin-2 particle with nonuniversal couplings in the Drell-Yan process, Phys. Rev. D 97 (2018) 094028 [arXiv: 1710.04184] [inSPIRE].

[16] N. Arkani-Hamed, S. Dimopoulos and G.R. Dvali, The hierarchy problem and new dimensions at a millimeter, Phys. Lett. B 429 (1998) 263 [hep-ph/9803315] [INSPIRE].

[17] T. Ahmed et al., NNLO QCD corrections to the Drell-Yan cross section in models of TeV-scale gravity, Eur. Phys. J. C 77 (2017) 22 [arXiv:1606.08454] [INSPIRE].

[18] G.F. Sterman, Summation of Large Corrections to Short Distance Hadronic Cross-Sections, Nucl. Phys. B 281 (1987) 310 [inSPIRE].

[19] S. Catani and L. Trentadue, Comment on QCD exponentiation at large x, Nucl. Phys. B 353 (1991) 183 [INSPIRE].

[20] S. Catani, D. de Florian, M. Grazzini and P. Nason, Soft gluon resummation for Higgs boson production at hadron colliders, JHEP 07 (2003) 028 [hep-ph/0306211] [INSPIRE].

[21] T.O. Eynck, E. Laenen and L. Magnea, Exponentiation of the Drell-Yan cross-section near partonic threshold in the DIS and MS-bar schemes, JHEP 06 (2003) 057 [hep-ph/0305179] [INSPIRE].

[22] S. Moch, J.A.M. Vermaseren and A. Vogt, Higher-order corrections in threshold resummation, Nucl. Phys. B 726 (2005) 317 [hep-ph/0506288] [INSPIRE].

[23] T. Becher, M. Neubert and B.D. Pecjak, Factorization and Momentum-Space Resummation in Deep-Inelastic Scattering, JHEP 01 (2007) 076 [hep-ph/0607228] [INSPIRE].

[24] S. Catani, L. Cieri, D. de Florian, G. Ferrera and M. Grazzini, Threshold resummation at $N^{3} L L$ accuracy and soft-virtual cross sections at $N^{3} L O$, Nucl. Phys. B 888 (2014) 75 [arXiv: 1405.4827] [INSPIRE].

[25] T. Ahmed, A.H. Ajjath, G. Das, P. Mukherjee, V. Ravindran and S. Tiwari, Soft-virtual correction and threshold resummation for n-colorless particles to fourth order in QCD: Part I, arXiv:2010.02979 [INSPIRE].

[26] S. Moch and A. Vogt, Higher-order soft corrections to lepton pair and Higgs boson production, Phys. Lett. B 631 (2005) 48 [hep-ph/0508265] [INSPIRE].

[27] D. de Florian and J. Zurita, Soft-gluon resummation for pseudoscalar Higgs boson production at hadron colliders, Phys. Lett. B 659 (2008) 813 [arXiv:0711.1916] [INSPIRE].

[28] W. Beenakker, S. Brensing, M. Krämer, A. Kulesza, E. Laenen and I. Niessen, Soft-gluon resummation for squark and gluino hadroproduction, JHEP 12 (2009) 041 [arXiv: 0909.4418] [INSPIRE]. 
[29] N. Kidonakis, NNLL resummation for s-channel single top quark production, Phys. Rev. D 81 (2010) 054028 [arXiv: 1001.5034] [INSPIRE].

[30] C.W. Bauer, F.J. Tackmann, J.R. Walsh and S. Zuberi, Factorization and Resummation for Dijet Invariant Mass Spectra, Phys. Rev. D 85 (2012) 074006 [arXiv:1106.6047] [InSPIRE].

[31] R.V. Harlander, A. Kulesza, V. Theeuwes and T. Zirke, Soft gluon resummation for gluon-induced Higgs Strahlung, JHEP 11 (2014) 082 [arXiv: 1410.0217] [INSPIRE].

[32] M. Bonvini and S. Marzani, Resummed Higgs cross section at $N^{3} L L$, JHEP 09 (2014) 007 [arXiv: 1405.3654] [INSPIRE].

[33] M. Bonvini, A.S. Papanastasiou and F.J. Tackmann, Resummation and matching of b-quark mass effects in $b \bar{b} H$ production, JHEP 11 (2015) 196 [arXiv:1508.03288] [INSPIRE].

[34] T. Ahmed, G. Das, M.C. Kumar, N. Rana and V. Ravindran, RG improved Higgs boson production to $N^{3} L O$ in $Q C D$, arXiv:1505.07422 [INSPIRE].

[35] A. Banfi et al., Jet-vetoed Higgs cross section in gluon fusion at $N^{3} L O+N N L L$ with small-R resummation, JHEP 04 (2016) 049 [arXiv: 1511.02886] [INSPIRE].

[36] T. Schmidt and M. Spira, Higgs Boson Production via Gluon Fusion: Soft-Gluon Resummation including Mass Effects, Phys. Rev. D 93 (2016) 014022 [arXiv:1509.00195] [INSPIRE].

[37] T. Ahmed et al., Pseudo-scalar Higgs boson production at $N^{3} L O_{A}+N^{3} L L^{\prime}$, Eur. Phys. J. C 76 (2016) 663 [arXiv:1606.00837] [INSPIRE].

[38] M. Bonvini, S. Marzani, C. Muselli and L. Rottoli, On the Higgs cross section at $N^{3} L O+N^{3} L L$ and its uncertainty, JHEP 08 (2016) 105 [arXiv: 1603.08000] [INSPIRE].

[39] A.H. Ajjath, A. Chakraborty, G. Das, P. Mukherjee and V. Ravindran, Resummed prediction for Higgs boson production through $b \overline{\mathrm{b}}$ annihilation at $N^{3} L L$, JHEP 11 (2019) 006 [arXiv: 1905.03771] [INSPIRE].

[40] M. Krämer, A. Kulesza, A. Mück and R. Schürmann, Threshold Resummation for Dark-Matter Production at the LHC, JHEP 07 (2019) 064 [arXiv:1903.06417] [INSPIRE].

[41] G. Das, S.-O. Moch and A. Vogt, Soft corrections to inclusive deep-inelastic scattering at four loops and beyond, JHEP 03 (2020) 116 [arXiv:1912.12920] [INSPIRE].

[42] A.H. Ajjath, G. Das, M.C. Kumar, P. Mukherjee, V. Ravindran and K. Samanta, Resummed Drell-Yan cross-section at $N^{3} L L$, JHEP 10 (2020) 153 [arXiv: 2001.11377] [INSPIRE].

[43] G. Das, S. Moch and A. Vogt, Approximate four-loop QCD corrections to the Higgs-boson production cross section, Phys. Lett. B 807 (2020) 135546 [arXiv:2004.00563] [INSPIRE].

[44] G. Das, M.C. Kumar and K. Samanta, Resummed inclusive cross-section in ADD model at $N^{3} L L$, JHEP 10 (2020) 161 [arXiv:1912.13039] [INSPIRE].

[45] G. Das, M.C. Kumar and K. Samanta, Resummed inclusive cross-section in Randall-Sundrum model at NNLO+NNLL, JHEP 07 (2020) 040 [arXiv:2004.03938] [INSPIRE].

[46] S.D. Joglekar and B.W. Lee, General Theory of Renormalization of Gauge Invariant Operators, Annals Phys. 97 (1976) 160 [INSPIRE].

[47] M. Henneaux, Remarks on the renormalization of gauge invariant operators in Yang-Mills theory, Phys. Lett. B 313 (1993) 35 [Erratum ibid. 316 (1993) 633] [hep-th/9306101] [INSPIRE]. 
[48] T. Ahmed, P. Banerjee, P.K. Dhani, P. Mathews, N. Rana and V. Ravindran, Three loop form factors of a massive spin-2 particle with nonuniversal coupling, Phys. Rev. D 95 (2017) 034035 [arXiv: 1612.00024] [INSPIRE].

[49] C. Anastasiou et al., Higgs boson gluon-fusion production at threshold in $N^{3} L O Q C D$, Phys. Lett. B 737 (2014) 325 [arXiv: 1403.4616] [INSPIRE].

[50] E. Laenen and L. Magnea, Threshold resummation for electroweak annihilation from DIS data, Phys. Lett. B 632 (2006) 270 [hep-ph/0508284] [INSPIRE].

[51] T. Ahmed, M. Mahakhud, N. Rana and V. Ravindran, Drell-Yan Production at Threshold to Third Order in QCD, Phys. Rev. Lett. 113 (2014) 112002 [arXiv: 1404.0366] [INSPIRE].

[52] Y. Li, A. von Manteuffel, R.M. Schabinger and H.X. Zhu, $N^{3} L O$ Higgs boson and Drell-Yan production at threshold: The one-loop two-emission contribution, Phys. Rev. D 90 (2014) 053006 [arXiv: 1404.5839] [INSPIRE].

[53] S. Moch, J.A.M. Vermaseren and A. Vogt, The three loop splitting functions in QCD: The nonsinglet case, Nucl. Phys. B 688 (2004) 101 [hep-ph/0403192] [InSPIRE].

[54] J. Henn, A.V. Smirnov, V.A. Smirnov, M. Steinhauser and R.N. Lee, Four-loop photon quark form factor and cusp anomalous dimension in the large- $N_{c}$ limit of QCD, JHEP 03 (2017) 139 [arXiv: 1612.04389] [INSPIRE].

[55] S. Moch, B. Ruijl, T. Ueda, J.A.M. Vermaseren and A. Vogt, Four-Loop Non-Singlet Splitting Functions in the Planar Limit and Beyond, JHEP 10 (2017) 041 [arXiv: 1707.08315] [INSPIRE].

[56] A. Grozin, Four-loop cusp anomalous dimension in QED, JHEP 06 (2018) 073 [Addendum ibid. 01 (2019) 134] [arXiv: 1805.05050] [INSPIRE].

[57] J.M. Henn, T. Peraro, M. Stahlhofen and P. Wasser, Matter dependence of the four-loop cusp anomalous dimension, Phys. Rev. Lett. 122 (2019) 201602 [arXiv: 1901.03693] [INSPIRE].

[58] R. Brüser, A. Grozin, J.M. Henn and M. Stahlhofen, Matter dependence of the four-loop QCD cusp anomalous dimension: from small angles to all angles, JHEP 05 (2019) 186 [arXiv: 1902.05076] [INSPIRE].

[59] J. Davies, A. Vogt, B. Ruijl, T. Ueda and J.A.M. Vermaseren, Large- $N_{f}$ contributions to the four-loop splitting functions in QCD, Nucl. Phys. B 915 (2017) 335 [arXiv:1610.07477] [INSPIRE].

[60] R.N. Lee, A.V. Smirnov, V.A. Smirnov and M. Steinhauser, The $n_{f}^{2}$ contributions to fermionic four-loop form factors, Phys. Rev. D 96 (2017) 014008 [arXiv:1705.06862] [INSPIRE].

[61] J.A. Gracey, Anomalous dimension of nonsinglet Wilson operators at $O\left(1 / N_{f}\right)$ in deep inelastic scattering, Phys. Lett. B 322 (1994) 141 [hep-ph/9401214] [INSPIRE].

[62] M. Beneke and V.M. Braun, Power corrections and renormalons in Drell-Yan production, Nucl. Phys. B 454 (1995) 253 [hep-ph/9506452] [INSPIRE].

[63] S. Moch, B. Ruijl, T. Ueda, J.A.M. Vermaseren and A. Vogt, On quartic colour factors in splitting functions and the gluon cusp anomalous dimension, Phys. Lett. B 782 (2018) 627 [arXiv: 1805.09638] [INSPIRE].

[64] R.N. Lee, A.V. Smirnov, V.A. Smirnov and M. Steinhauser, Four-loop quark form factor with quartic fundamental colour factor, JHEP 02 (2019) 172 [arXiv: 1901.02898] [INSPIRE]. 
[65] J.M. Henn, G.P. Korchemsky and B. Mistlberger, The full four-loop cusp anomalous dimension in $\mathcal{N}=4$ super Yang-Mills and QCD, JHEP 04 (2020) 018 [arXiv:1911.10174] [INSPIRE].

[66] T. Huber, A. von Manteuffel, E. Panzer, R.M. Schabinger and G. Yang, The four-loop cusp anomalous dimension from the $N=4$ Sudakov form factor, Phys. Lett. B 807 (2020) 135543 [arXiv: 1912.13459] [INSPIRE].

[67] A. von Manteuffel, E. Panzer and R.M. Schabinger, Cusp and collinear anomalous dimensions in four-loop QCD from form factors, Phys. Rev. Lett. 124 (2020) 162001 [arXiv: 2002.04617] [INSPIRE].

[68] S. Catani, M.L. Mangano, P. Nason and L. Trentadue, The resummation of soft gluons in hadronic collisions, Nucl. Phys. B 478 (1996) 273 [hep-ph/9604351] [INSPIRE].

[69] S. Forte, G. Ridolfi, J. Rojo and M. Ubiali, Borel resummation of soft gluon radiation and higher twists, Phys. Lett. B 635 (2006) 313 [hep-ph/0601048] [INSPIRE].

[70] R. Abbate, S. Forte and G. Ridolfi, A new prescription for soft gluon resummation, Phys. Lett. B 657 (2007) 55 [arXiv:0707.2452] [INSPIRE].

[71] M. Bonvini, Resummation of soft and hard gluon radiation in perturbative QCD, Ph.D. thesis, Genoa University (2012), arXiv:1212.0480 [INSPIRE].

[72] L. Magnea and G.F. Sterman, Analytic continuation of the Sudakov form-factor in QCD, Phys. Rev. D 42 (1990) 4222 [inSPIRE].

[73] S.M. Aybat, L.J. Dixon and G.F. Sterman, The two-loop soft anomalous dimension matrix and resummation at next-to-next-to leading pole, Phys. Rev. D 74 (2006) 074004 [hep-ph/0607309] [INSPIRE].

[74] L.J. Dixon, L. Magnea and G.F. Sterman, Universal structure of subleading infrared poles in gauge theory amplitudes, JHEP 08 (2008) 022 [arXiv:0805.3515] [INSPIRE].

[75] J. Butterworth et al., PDF4LHC recommendations for LHC Run II, J. Phys. G 43 (2016) 023001 [arXiv: 1510.03865] [INSPIRE].

[76] A. Buckley et al., LHAPDF6: parton density access in the LHC precision era, Eur. Phys. J. C 75 (2015) 132 [arXiv:1412.7420] [INSPIRE].

[77] ATLAS collaboration, Search for new phenomena in high-mass diphoton final states using $37 \mathrm{fb}^{-1}$ of proton-proton collisions collected at $\sqrt{s}=13 \mathrm{TeV}$ with the ATLAS detector, Phys. Lett. B 775 (2017) 105 [arXiv:1707.04147] [INSPIRE].

[78] M. Botje et al., The PDF4LHC Working Group Interim Recommendations, arXiv: 1101.0538 [INSPIRE].

[79] L.A. Harland-Lang, A.D. Martin, P. Motylinski and R.S. Thorne, Parton distributions in the LHC era: MMHT 2014 PDFs, Eur. Phys. J. C 75 (2015) 204 [arXiv:1412.3989] [InSPIRE].

[80] S. Dulat et al., New parton distribution functions from a global analysis of quantum chromodynamics, Phys. Rev. D 93 (2016) 033006 [arXiv:1506.07443] [InSPIRE].

[81] S. Alekhin, J. Blumlein and S. Moch, The ABM parton distributions tuned to LHC data, Phys. Rev. D 89 (2014) 054028 [arXiv:1310.3059] [INSPIRE].

[82] NNPDF collaboration, Parton distributions from high-precision collider data, Eur. Phys. J. $C 77$ (2017) 663 [arXiv:1706.00428] [INSPIRE]. 
[83] T. van Ritbergen, J.A.M. Vermaseren and S.A. Larin, The four loop $\beta$-function in quantum chromodynamics, Phys. Lett. B 400 (1997) 379 [hep-ph/9701390] [INSPIRE].

[84] M. Cacciari and N. Houdeau, Meaningful characterisation of perturbative theoretical uncertainties, JHEP 09 (2011) 039 [arXiv:1105.5152] [INSPIRE].

[85] M. Bonvini, Probabilistic definition of the perturbative theoretical uncertainty from missing higher orders, Eur. Phys. J. C 80 (2020) 989 [arXiv: 2006.16293] [INSPIRE]. 